\title{
Synchronization of Switched Complex Bipartite Neural Networks with Infinite Distributed Delays and Derivative Coupling
}

\author{
Qiuxiang Bian, ${ }^{1}$ Jinde Cao, ${ }^{2,3}$ Jie Wu, ${ }^{1}$ Hongxing Yao, ${ }^{4}$ Tingfang Zhang, ${ }^{1}$ and Xiaoxu Ling ${ }^{5}$ \\ ${ }^{1}$ Department of Mathematics and Physics, Jiangsu University of Science and Technology, Zhenjiang 212003, China \\ ${ }^{2}$ Department of Mathematics, Southeast University, Nanjing 210096, China \\ ${ }^{3}$ Department of Mathematics, King Abdulaziz University, Jeddah 21589, Saudi Arabia \\ ${ }^{4}$ Department of Mathematics, Jiangsu University, Zhenjiang 212003, China \\ ${ }^{5}$ Faculty of Economics and Management, Nanjing University of Aeronautics \& Astronautics, Nanjing 212096, China
}

Correspondence should be addressed to Qiuxiang Bian; bianqx_1@163.com

Received 7 March 2013; Accepted 8 April 2013

Academic Editor: Hongli Dong

Copyright (C) 2013 Qiuxiang Bian et al. This is an open access article distributed under the Creative Commons Attribution License, which permits unrestricted use, distribution, and reproduction in any medium, provided the original work is properly cited.

\begin{abstract}
A new model of switched complex bipartite neural network (SCBNN) with infinite distributed delays and derivative coupling is established. Using linear matrix inequality (LMI) approach, some synchronization criteria are proposed to ensure the synchronization between two SCBNNs by constructing effective controllers. Some numerical simulations are provided to illustrate the effectiveness of the theoretical results obtained in this paper.
\end{abstract}

\section{Introduction}

In recent years, neural networks have been intensively studied due to their potential applications in many different areas such as signal and image processing, content-addressable memory, optimization, and parallel computation [1-3]. Bidirectional associative memory (BAM) neural networks were first proposed by Kosko in [4, 5]. This class of networks has good applications in pattern recognition, solving optimization problems, and automatic control engineering. A large number of results on the dynamical behavior of BAM neural networks have been reported [6-9].

Switched systems, as an important kind of hybrid systems, have drawn considerable attention of researchers because of their theoretical significance and practical applications [1012]. Switched systems are composed of a family of continuous-time or discrete-time subsystems and a rule that specifies the switching among them $[13,14]$. Recently, the switched neural networks, whose individual subsystems are a set of neural networks, have found applications in the field of high speed signal processing, artificial intelligence, and biology, so there are many theoretical results about the switched neural networks [15-17].

Complex networks, which are a set of interconnected nodes with specific dynamics, have sparked the interest of many researchers from various fields of science and engineering such as the World Wide Web, electrical power grids, global economic markets, sensor networks; for example, see [18-20] and references therein. Bipartite networks are an important kind of complex networks, whose nodes can be divided into two disjoint nonempty sets such that every edge only connects a pair of nodes, which belong to different sets. Many real-world networks are naturally bipartite networks, such as the papers-scientists networks [21] and producerconsumer networks [22]. Recently, authors [23] have introduced a bipartite-graph complex dynamical network model that is only linearly coupled and has no delays. It is well known that time delays exist commonly in real-world systems. Therefore, many models of coupled networks with coupling delays are proposed, for example, constant single time delay [24], time-varying delays [25], and mix-time delays [26]. On the other hand, the coupled network often occurs 
in other forms, for example, nonlinearly coupled networks [27] and linearly derivative coupled networks [28]. In [29], a general model of bipartite dynamical network (BDN) with distributed delays and nonlinear derivative coupling was introduced. Synchronization of complex networks has been intensively investigated since they can be applied in power system control, secure communication, automatic control, chemical reaction, and so on [30-32]. The study of synchronization of coupled neural networks is an important step for both understanding brain science and designing coupled neural networks for practical use. Yu et al. [33] consider the synchronization of switched linearly coupled neural networks with constant delays, but the controllers are complex and changed with the switched rule. Synchronization of two coupled BDNs was investigated by adaptive method [29], but the controllers are complicated and the model does not include infinite distributed delays coupling and switching. Extending BAM neural networks to complex networks, we get complex bipartite dynamical networks (CBDNs). The dynamics of individual node in CBDNs is switched system and the switched coupling is considered; switched complex bipartite neural network (SCBNN) can be obtained. To the best of our knowledge, up to now, there is not any work that discusses the synchronization problem in SCBNN.

Motivated by the previous discussion, we first proposed a model of SCBNN, and then investigated the synchronization between two SCBNNs with infinite distributed delays and derivative coupling. Using adaptive controllers and linear matrix inequality (LMI) approach, some synchronization criteria are proposed to ensure the synchronization between two coupled SCBNNs. In our paper, the proposed controllers are simpler and do not change with the switched rule, which can be realize more easily.

The paper is organized as follows. In Section 2, a model of SCBNN with infinite distributed delays and derivative coupling is presented, and some hypotheses and lemmas are given too. In Section 3, several synchronization criteria on the SCBNNs are deduced. In Section 4, numerical examples are given to demonstrate the effectiveness of the proposed controller design methods in Section 3. Finally, conclusions are given in Section 5.

Notations. Throughout this paper, $\rho_{\max }(\cdot)$ and $\rho_{\min }(\cdot)$ denote the maximum eigenvalue and minimum eigenvalue of a real symmetric matrix, respectively. The notation $*$ denotes the symmetric block.

\section{Model Description, Assumptions, and Lemmas}

Consider a complex bipartite dynamical network (CBDN) consisting of two disjoint nonempty node sets $V_{1}$ and $V_{2}$. Suppose that $V_{1}=\left\{\mu_{1}, \mu_{2}, \ldots, \mu_{l}\right\}$ and $V_{2}=\left\{v_{1}, \nu_{2}, \ldots, v_{m}\right\}$, $l, m$ are integer. The coupled network is described as follows:

$$
\begin{aligned}
\dot{x}_{i}(t)= & -D x_{i}+R_{1} f_{1}\left(x_{i}(t)\right)+R_{2} f_{2}\left(x_{i}(t-\tau(t))\right)+I \\
& +\sum_{j=1}^{m} a_{i j} y_{j}\left(t-\tau_{1}(t)\right)+\sum_{j=1}^{m} b_{i j} g\left(\dot{y}_{j}\left(t-\tau_{2}(t)\right)\right)
\end{aligned}
$$

$$
\begin{aligned}
& +\sum_{j=1}^{m} c_{i j} \int_{-\infty}^{t} h(t-s) k\left(y_{j}(s)\right) \mathrm{d} s, \\
i=1,2, \ldots, l, & \\
\dot{y}_{j}(t)= & -\bar{D} y_{j}+\bar{R}_{1} \bar{f}_{1}\left(y_{j}(t)\right)+\bar{R}_{2} \bar{f}_{2}\left(y_{j}(t-\sigma(t))\right)+J \\
& +\sum_{i=1}^{l} \bar{a}_{j i} x_{i}\left(t-\sigma_{1}(t)\right)+\sum_{i=1}^{l} \bar{b}_{j i} \bar{g}\left(\dot{x}_{i}\left(t-\sigma_{2}(t)\right)\right) \\
& +\sum_{i=1}^{l} \bar{c}_{j i} \int_{-\infty}^{t} \bar{h}(t-s) \bar{k}\left(x_{i}(s)\right) \mathrm{d} s, \quad j=1,2, \ldots, m,
\end{aligned}
$$

where $x_{i}(t)=\left(x_{i 1}(t), x_{i 2}(t), \ldots, x_{i n}(t)\right)^{T}, y_{j}(t)=\left(y_{j 1}(t)\right.$, $\left.y_{j 2}(t), \ldots, y_{j n}(t)\right)^{T} \in \mathrm{R}^{n}$ denotes the state variables of nodes $\mu_{i}$ and $\nu_{j}$, respectively. $D=\operatorname{diag}\left(d_{1}, d_{2}, \ldots, d_{n}\right)$ and $\bar{D}=$ $\operatorname{diag}\left(\bar{d}_{1}, \bar{d}_{2}, \ldots, \bar{d}_{n}\right)$ are diagonal matrices with $d_{i}, \bar{d}_{i}>0$. $R_{1}, \bar{R}_{1} \in \mathrm{R}^{n \times n}$ are weight matrices, $R_{2}, \bar{R}_{2} \in \mathrm{R}^{n \times n}$ are delayed weight matrices, $f_{k}\left(x_{i}\right)=\left(f_{k 1}\left(x_{i 1}\right), f_{k 2}\left(x_{i 2}\right), \ldots, f_{k n}\left(x_{i n}\right)\right)^{T}$, $\bar{f}_{k}\left(y_{j}\right)=\left(\bar{f}_{k 1}\left(y_{j 1}\right), \bar{f}_{k 2}\left(y_{j 2}\right), \ldots, \bar{f}_{k n}\left(y_{j n}\right)\right)^{T} \in \mathrm{R}^{n}, k=1,2$, $g\left(\dot{y}_{j}\right)=\left(g_{1}\left(\dot{y}_{j 1}\right), g_{2}\left(\dot{y}_{j 2}\right), \ldots, g_{n}\left(\dot{y}_{j n}\right)\right)^{T}, \bar{g}\left(\dot{x}_{i}\right)=\left(\bar{g}_{1}\left(\dot{x}_{i 1}\right)\right.$, $\left.\bar{g}_{2}\left(\dot{x}_{i 2}\right), \ldots, \bar{g}_{n}\left(\dot{x}_{i n}\right)\right)^{T}, k\left(y_{j}\right)=\left(k_{1}\left(y_{j 1}\right), k_{2}\left(y_{j 2}\right), \ldots, k_{n}\left(y_{j n}\right)\right)^{T}$, $\bar{k}\left(x_{i}\right)=\left(\bar{k}_{1}\left(x_{i 1}\right), \bar{k}_{2}\left(x_{i 2}\right), \ldots, \bar{k}_{n}\left(x_{i n}\right)\right)^{T} \in \mathrm{R}^{n}$ corresponds to the boundedness activation functions of neurons. $h(t)=\operatorname{diag}\left(h_{1}(t), h_{2}(t), \ldots, h_{n}(t)\right), \bar{h}(t)=\operatorname{diag}\left(\bar{h}_{1}(t), \bar{h}_{2}(t)\right.$, $\left.\ldots, \bar{h}_{n}(t)\right) \in \mathrm{R}^{n \times n}$ are the delay kernel functions. $\tau(t), \tau_{1}(t)$, $\tau_{2}(t), \sigma(t), \sigma_{1}(t)$, and $\sigma_{2}(t)>0$ are time delays. $\int_{-\infty}^{t} h(t-$ $s) k\left(y_{j}(s)\right) \mathrm{d} s$ and $\int_{-\infty}^{t} \bar{h}(t-s) \bar{k}\left(x_{i}(s)\right) \mathrm{d} s$ express infinite distributed delays. $I=\left(I^{1}, I^{2}, \ldots, I^{n}\right)^{T}$ and $J=\left(J^{1}, J^{2}, \ldots\right.$, $\left.J^{n}\right)^{T} \in \mathrm{R}^{n}$ are the constant external input vectors. The matrix $A=\left(a_{i j}\right)_{l \times m}$ is the delayed weight coupling matrix denoting coupling strength between nodes. If there is a connection from node $\mu_{i}$ to $v_{j}$, then $a_{i j} \neq 0$; otherwise, $a_{i j}=0$ and the matrix $A$ satisfies the sum of every row being zero. The definitions of the other coupling matrixes $B=\left(b_{i j}\right)_{l \times m}, C=$ $\left(c_{i j}\right)_{l \times m}, \bar{A}=\left(\bar{a}_{j i}\right)_{m \times l}, \bar{B}=\left(\bar{b}_{j i}\right)_{m \times l}$, and $\bar{C}=\left(\bar{c}_{j i}\right)_{m \times l}$ are similar to that of matrix $A$; hence, they are omitted here.

In this paper, we consider a class of switched complex bipartite neural network with infinite distributed delays and derivative coupling, which is described as follows:

$$
\begin{aligned}
& \dot{x}_{i}(t)=-D_{\lambda} x_{i}+R_{\lambda 1} f_{1}\left(x_{i}(t)\right)+R_{\lambda 2} f_{2}\left(x_{i}(t-\tau(t))\right) \\
&+I_{\lambda}+\sum_{j=1}^{m} a_{\lambda i j} y_{j}\left(t-\tau_{1}(t)\right) \\
&+\sum_{j=1}^{m} b_{\lambda i j} g\left(\dot{y}_{j}\left(t-\tau_{2}(t)\right)\right) \\
&+\sum_{j=1}^{m} c_{\lambda i j} \int_{-\infty}^{t} h(t-s) k\left(y_{j}(s)\right) \mathrm{d} s, \\
& i=1,2, \ldots, l,
\end{aligned}
$$




$$
\begin{aligned}
\dot{y}_{j}(t)= & -\bar{D}_{\lambda} y_{j}+\bar{R}_{\lambda 1} \bar{f}_{1}\left(y_{j}(t)\right)+\bar{R}_{\lambda 2} \bar{f}_{2}\left(y_{j}(t-\sigma(t))\right) \\
& +J_{\lambda}+\sum_{i=1}^{l} \bar{a}_{\lambda j i} x_{i}\left(t-\sigma_{1}(t)\right) \\
& +\sum_{i=1}^{l} \bar{b}_{\lambda j i} \bar{g}\left(\dot{x}_{i}\left(t-\sigma_{2}(t)\right)\right) \\
& +\sum_{i=1}^{l} \bar{c}_{\lambda j i} \int_{-\infty}^{t} \bar{h}(t-s) \bar{k}\left(x_{i}(s)\right) \mathrm{d} s, \quad j=1,2, \ldots, m,
\end{aligned}
$$

where switching signal $\lambda$ is piecewise constant functions, which is a value in the finite set $\aleph=\{1,2, \ldots, N\}$. This means that the matrices $\left\{D_{\lambda}, R_{\lambda 1}, R_{\lambda 2}, A_{\lambda}=\left(a_{\lambda i j}\right), B_{\lambda}=\right.$ $\left(b_{\lambda i j}\right), C_{\lambda}=\left(c_{\lambda i j}\right), I_{\lambda}, \bar{D}_{\lambda}, \bar{R}_{\lambda 1}, \bar{R}_{\lambda 2}, \bar{A}_{\lambda}=\left(\bar{a}_{\lambda j i}\right), \bar{B}_{\lambda}=\left(\bar{b}_{\lambda j i}\right)$, and $\left.\bar{C}_{\lambda}=\left(\bar{c}_{\lambda j i}\right), J_{\lambda}\right\}$ are allowed to take values at particular time, in a finite set $\left\{\left(D_{r}, R_{r 1}, R_{r 2}, A_{r}, B_{r}, C_{r}, I_{r}\right.\right.$, $\left.\left.\bar{D}_{r}, \bar{R}_{r 1}, \bar{R}_{r 2}, \bar{A}_{r}, \bar{B}_{r}, \bar{C}_{r}, J_{r}\right) \mid r=1,2, \ldots, N\right\}$. We define the function as follows:

$$
\begin{aligned}
& \xi_{r}(t, \lambda) \\
& = \begin{cases}1, & \text { when the switched system is described } \\
0, & \text { by the } r \text { th mode, that is, } \lambda=r,\end{cases}
\end{aligned}
$$

It follows that under any switching rules $\sum_{r=1}^{N} \xi_{r}(t, \lambda)=1$. Model (2) can be written as

$$
\begin{aligned}
\dot{x}_{i}(t)=\sum_{r=1}^{N} \xi_{r}( & t, \lambda) \\
\times & -D_{r} x_{i}+R_{r 1} f_{1}\left(x_{i}(t)\right) \\
& +R_{r 2} f_{2}\left(x_{i}(t-\tau(t))\right) \\
& +I_{r}+\sum_{j=1}^{m} a_{r i j} y_{j}\left(t-\tau_{1}(t)\right) \\
& +\sum_{j=1}^{m} b_{r i j} g\left(\dot{y}_{j}\left(t-\tau_{2}(t)\right)\right) \\
& \left.+\sum_{j=1}^{m} c_{r i j} \int_{-\infty}^{t} h(t-s) k\left(y_{j}(s)\right) \mathrm{d} s\right], \\
& \quad i=1,2, \ldots, l,
\end{aligned}
$$

$$
\begin{aligned}
\dot{y}_{j}(t)=\sum_{r=1}^{N} \xi_{r}( & t, \lambda) \\
\times[ & -\bar{D}_{r} y_{j}+\bar{R}_{r 1} \bar{f}_{1}\left(y_{j}(t)\right) \\
& +\bar{R}_{r 2} \bar{f}_{2}\left(y_{j}(t-\sigma(t))\right) \\
& +J_{r}+\sum_{i=1}^{l} \bar{a}_{r j i} x_{i}\left(t-\sigma_{1}(t)\right) \\
& +\sum_{i=1}^{l} \bar{b}_{r j i} \bar{g}\left(\dot{x}_{i}\left(t-\sigma_{2}(t)\right)\right) \\
& \left.+\sum_{i=1}^{l} \bar{c}_{r j i} \int_{-\infty}^{t} \bar{h}(t-s) \bar{k}\left(x_{i}(s)\right) \mathrm{d} s\right],
\end{aligned}
$$

The response network of the drive network (4) is

$$
\begin{aligned}
& \dot{\hat{x}}_{i}(t)=\sum_{r=1}^{N} \xi_{r}(t, \lambda) \\
& \times\left[-D_{r} \widehat{x}_{i}(t)+R_{r 1} f_{1}\left(\widehat{x}_{i}(t)\right)\right. \\
& +R_{r 2} f_{2}\left(\widehat{x}_{i}(t-\tau(t))\right) \\
& +I_{r}+\sum_{j=1}^{m} a_{r i j} \widehat{y}_{j}\left(t-\tau_{1}(t)\right) \\
& +\sum_{j=1}^{m} b_{r i j} g\left(\dot{\hat{y}}_{j}\left(t-\tau_{2}(t)\right)\right) \\
& \left.+\sum_{j=1}^{m} c_{r i j} \int_{-\infty}^{t} h(t-s) k\left(\widehat{y}_{j}(s)\right) \mathrm{d} s+u_{i}(t)\right], \\
& \dot{\hat{y}}_{j}(t)=\sum_{r=1}^{N} \xi_{r}(t, \lambda) \\
& \times\left[-\bar{D}_{r} \widehat{y}_{j}(t)+\bar{R}_{r 1} \bar{f}_{1}\left(\widehat{y}_{j}(t)\right)\right. \\
& +\bar{R}_{r 2} \bar{f}_{2}\left(\widehat{y}_{j}(t-\sigma(t))\right) \\
& +J_{r}+\sum_{i=1}^{l} \bar{a}_{r j i} \widehat{x}_{i}\left(t-\sigma_{1}(t)\right) \\
& +\sum_{i=1}^{l} \bar{b}_{r j i} \bar{g}\left(\dot{\hat{x}}_{i}\left(t-\sigma_{2}(t)\right)\right) \\
& \left.+\sum_{i=1}^{l} \bar{c}_{r j i} \int_{-\infty}^{t} \bar{h}(t-s) \bar{k}\left(\hat{x}_{i}(s)\right) \mathrm{d} s+v_{j}(t)\right],
\end{aligned}
$$

where $u_{i}(t)$ and $v_{j}(t) \in R^{n}$ are the control inputs. 
Let $e_{i}(t)=\widehat{x}_{i}(t)-x_{i}(t), \varepsilon_{j}(t)=\widehat{y}_{j}(t)-y_{j}(t), i=1,2, \ldots, l$, and $j=1,2, \ldots, m$. The error dynamical system of (4) and (5) is given by

$$
\begin{aligned}
\dot{e}_{i}(t)=\sum_{r=1}^{N} \xi_{r}(t, \lambda) & \\
\times & {\left[-D_{r} e_{i}(t)+R_{r 1} F_{1}\left(e_{i}(t)\right)\right.} \\
& +R_{r 2} F_{2}\left(e_{i}(t-\tau(t))\right) \\
& +\sum_{j=1}^{m} a_{r i j} \varepsilon_{j}\left(t-\tau_{1}(t)\right) \\
& +\sum_{j=1}^{m} b_{r i j} G\left(\dot{\varepsilon}_{j}\left(t-\tau_{2}(t)\right)\right) \\
& \left.+\sum_{j=1}^{m} c_{r i j} \int_{-\infty}^{t} h(t-s) K\left(\varepsilon_{j}(s)\right) \mathrm{d} s+u_{i}(t)\right], \\
& i=1,2, \ldots, l,
\end{aligned}
$$$$
\dot{\varepsilon}_{j}(t)=\sum_{r=1}^{N} \xi_{r}(t, \lambda)
$$$$
\times\left[-\bar{D}_{r} \varepsilon_{j}(t)+\bar{R}_{r 1} \bar{F}_{1}\left(\varepsilon_{j}(t)\right)\right.
$$$$
+\bar{R}_{r 2} \bar{F}_{2}\left(\varepsilon_{j}(t-\sigma(t))\right)
$$$$
+\sum_{i=1}^{l} \bar{a}_{r j i} e_{i}\left(t-\sigma_{1}(t)\right)
$$$$
+\sum_{i=1}^{l} \bar{b}_{r j i} \bar{G}\left(\dot{e}_{i}\left(t-\sigma_{2}(t)\right)\right)
$$$$
\left.+\sum_{i=1}^{l} \bar{c}_{r j i} \int_{-\infty}^{t} \bar{h}(t-s) \bar{K}\left(e_{i}(s)\right) \mathrm{d} s+v_{j}(t)\right],
$$$$
j=1,2, \ldots, m \text {, }
$$

where

$$
\begin{gathered}
F_{k}\left(e_{i}(t)\right)=f_{k}\left(\hat{x}_{i}(t)\right)-f_{k}\left(x_{i}(t)\right), \\
\bar{F}_{k}\left(\varepsilon_{j}(t)\right)=\bar{f}_{k}\left(\hat{y}_{j}(t)\right)-\bar{f}_{k}\left(y_{j}(t)\right), \quad k=1,2, \\
G\left(\dot{\varepsilon}_{j}(t)\right)=g\left(\dot{\hat{y}}_{j}(t)\right)-g\left(\dot{y}_{j}(t)\right), \\
\bar{G}\left(\dot{e}_{i}(t)\right)=\bar{g}\left(\dot{\hat{x}}_{i}(t)\right)-\bar{g}\left(\dot{x}_{i}(t)\right),
\end{gathered}
$$

$$
\begin{aligned}
K\left(\varepsilon_{j}(s)\right) & =k\left(\hat{y}_{j}(s)\right)-k\left(y_{j}(s)\right) \\
& =\left(K_{1}\left(\varepsilon_{j 1}(s)\right), \ldots, K_{n}\left(\varepsilon_{j n}(s)\right)\right)^{T}, \\
\bar{K}\left(e_{i}(s)\right) & =\bar{k}\left(\widehat{x}_{i}(s)\right)-\bar{k}\left(x_{i}(s)\right) \\
& =\left(\bar{K}_{1}\left(e_{i 1}(s)\right), \ldots, \bar{K}_{n}\left(e_{i n}(s)\right)\right)^{T} .
\end{aligned}
$$

In this paper, the following assumptions and lemmas are needed.

$\left(S_{1}\right)$ There exist diagonal matrices $L_{i}^{-}=\operatorname{diag}\left(l_{i 1}^{-}, l_{i 2}^{-}, \ldots, l_{\text {in }}^{-}\right)$ and $L_{i}^{+}=\operatorname{diag}\left(l_{i 1}^{+}, l_{i 2}^{+}, \ldots, l_{i n}^{+}\right)$, such that

$$
\begin{gathered}
l_{k j}^{-} \leq \frac{f_{k j}(x)-f_{k j}(y)}{x-y} \leq l_{k j}^{+}, \quad l_{3 j}^{-} \leq \frac{k_{j}(x)-k_{j}(y)}{x-y} \leq l_{3 j}^{+}, \\
l_{4 j}^{-} \leq \frac{g_{j}(x)-g_{j}(y)}{x-y} \leq l_{4 j}^{+},
\end{gathered}
$$

$\forall x, y \in \mathrm{R}$ and $x \neq y, i=1,2,3,4, j=1,2, \ldots, n$, and $k=1,2$.

$\left(S_{2}\right)$ There exist diagonal matrices $\bar{L}_{i}^{-}=\operatorname{diag}\left(\bar{l}_{i 1}^{-} \bar{l}_{i 2}^{-}, \ldots\right.$, $\left.\bar{l}_{i n}^{-}\right)$and $\bar{L}_{i}^{+}=\operatorname{diag}\left(\bar{l}_{i 1}^{+}, \bar{l}_{i 2}^{+}, \ldots, \bar{l}_{i n}^{+}\right)$, such that

$$
\begin{gathered}
\bar{l}_{k j}^{-} \leq \frac{\bar{f}_{k j}(x)-\bar{f}_{k j}(y)}{x-y} \leq \bar{l}_{k j}^{+}, \quad \bar{l}_{3 j}^{-} \leq \frac{\bar{k}_{j}(x)-\bar{k}_{j}(y)}{x-y} \leq \bar{l}_{3 j}^{+}, \\
\bar{l}_{4 j}^{-} \leq \frac{\bar{g}_{j}(x)-\bar{g}_{j}(y)}{x-y} \leq \bar{l}_{4 j}^{+},
\end{gathered}
$$

$\forall x, y \in \mathrm{R}$ and $x \neq y, i=1,2,3,4, j=1,2, \ldots, n$, and $k=1,2$.

$\left(S_{3}\right) \tau(t), \tau_{1}(t), \tau_{2}(t), \sigma(t), \sigma_{1}(t)$, and $\sigma_{2}(t)$ are differential functions with $\dot{\tau}(t)<\tau<1, \dot{\sigma}(t)<\sigma<1, \dot{\tau}_{1}(t)<\tau_{1}<$ $1, \dot{\sigma}_{1}(t)<\sigma_{1}<1, \dot{\tau}_{2}(t)<\tau_{2}<1$, and $\dot{\sigma}_{2}(t)<\sigma_{2}<1$.

$\left(S_{4}\right) h_{i}(t), \bar{h}_{i}(t)$ are real-value nonnegative continuous functions defined in $[0, \infty)$ satisfying

$$
\int_{0}^{\infty} h_{i}(s) \mathrm{d} s<\infty, \quad \int_{0}^{\infty} \bar{h}_{i}(s) \mathrm{d} s<\infty, \quad i=1,2, \ldots, n .
$$

Lemma 1 (see [34]). Given any real matrices $\Sigma_{1}, \Sigma_{2}$, and $\Sigma_{3}$ of appropriate dimensions and a scalar $\varepsilon>0$ such that $0<$ $\Sigma_{3}=\Sigma_{3}^{T}$, then the following inequality holds:

$$
\Sigma_{1}^{T} \Sigma_{2}+\Sigma_{2}^{T} \Sigma_{1} \leq \varepsilon \Sigma_{1}^{T} \Sigma_{3} \Sigma_{1}+\varepsilon^{-1} \Sigma_{2}^{T} \Sigma_{3}^{-1} \Sigma_{2} .
$$

Lemma 2 (see [35]). Given a positive definite matrix $P \in$ $R^{n \times n}$ and a symmetric matrix $Q \in R^{n \times n}$, then

$$
\begin{array}{r}
\rho_{\text {min }}\left(P^{-1} Q\right) x^{T} P x \leq x^{T} Q x \leq \rho_{\max }\left(P^{-1} Q\right) x^{T} P x, \\
\forall x \in R^{n} .
\end{array}
$$


Lemma 3 (Schur complement). Given constant symmetric matrices $\Sigma_{1}, \Sigma_{2}$, and $\Sigma_{3}$, where $\Sigma_{1}=\Sigma_{1}^{T}$ and $0<\Sigma_{2}=\Sigma_{2}^{T}$, then $\Sigma_{1}+\Sigma_{3}^{T} \Sigma_{2}^{-1} \Sigma_{3}<0$ if and only if

$$
\left(\begin{array}{cc}
\Sigma_{1} & \Sigma_{3}^{T} \\
\Sigma_{3} & -\Sigma_{2}
\end{array}\right)<0 \quad \text { or } \quad\left(\begin{array}{cc}
-\Sigma_{2} & \Sigma_{3} \\
\Sigma_{3}^{T} & \Sigma_{1}
\end{array}\right)<0
$$

For convenience, let

$$
\begin{gathered}
L_{i}=\operatorname{diag}\left(\max \left\{\left|l_{i 1}^{-}\right|,\left|l_{i 1}^{+}\right|\right\},\right. \\
\left.\max \left\{\left|l_{i 2}^{-}\right|,\left|l_{i 2}^{+}\right|\right\}, \ldots, \max \left\{\left|l_{i n}^{-}\right|,\left|l_{i n}^{+}\right|\right\}\right), \\
i=1,2,3,4, \\
\bar{L}_{i}=\operatorname{diag}\left(\max \left\{\left|\bar{l}_{i 1}^{-}\right|,\left|\bar{l}_{i 1}^{+}\right|\right\},\right. \\
\left.\max \left\{\left|\bar{l}_{i 2}^{-}\right|,\left|\bar{l}_{i 2}^{+}\right|\right\}, \ldots, \max \left\{\left|\bar{l}_{i n}^{-}\right|,\left|\bar{l}_{i n}^{+}\right|\right\}\right), \\
i=1,2,3,4, \\
H=\operatorname{diag}\left(\int_{0}^{\infty} h_{1}(v) \mathrm{d} v, \int_{0}^{\infty} h_{2}(v) \mathrm{d} v, \ldots, \int_{0}^{\infty} h_{n}(v) \mathrm{d} v\right), \\
\bar{H}=\operatorname{diag}\left(\int_{0}^{\infty} \bar{h}_{1}(v) \mathrm{d} v, \int_{0}^{\infty} \bar{h}_{2}(v) \mathrm{d} v, \ldots, \int_{0}^{\infty} \bar{h}_{n}(v) \mathrm{d} v\right) .
\end{gathered}
$$

\section{Main Results}

Theorem 4. Under assumptions $\left(S_{1}\right)-\left(S_{4}\right)$, the two coupled SCBNNs (4) and (5) can be synchronized, if there exist positive constants, $\alpha, \beta, p, \bar{p}, \gamma_{i}, \eta_{j}(i=1,2, \ldots, l, j=1,2, \ldots, m)$, $n \times n$ positive matrices $P, Q, U, \bar{P}, \bar{Q}, \bar{U}$ and $n \times n$ diagonal positive matrices $W=\operatorname{diag}\left(w_{1}, w_{2}, \ldots, w_{n}\right), \bar{W}=$ $\operatorname{diag}\left(\bar{w}_{1}, \bar{w}_{2}, \ldots, \bar{w}_{n}\right), M_{i}, \bar{M}_{i}(i=1,2,3)$ such that

$$
\left.\begin{array}{cccccc}
Z_{r i} & P R_{r 1} & P R_{r 2} & \left(\sum_{j=1}^{m} a_{r i j}^{2}\right)^{1 / 2} P & \left(\sum_{j=1}^{m} b_{r i j}^{2}\right)^{1 / 2} P & \left(l \sum_{j=1}^{m} c_{r i j}^{2}\right)^{1 / 2} P \\
* & -M_{1} & 0 & 0 & 0 & 0 \\
* & * & -M_{2} & 0 & 0 & 0 \\
* & * & * & -M_{3} & 0 & 0 \\
* & * & * & * & -I_{n} & 0 \\
* & * & * & * & * & -W
\end{array}\right]
$$

$$
\left.\begin{array}{cccccc}
\bar{Z}_{r j} & \bar{P} \bar{R}_{r 1} & \bar{P} \bar{R}_{r 2} & \left(\sum_{i=1}^{l} \bar{a}_{r j i}^{2}\right)^{1 / 2} \bar{P} & \left(\sum_{i=1}^{l} \bar{b}_{r j i}^{2}\right)^{1 / 2} \bar{P} & \left(m \sum_{i=1}^{l} \bar{c}_{r j i}^{2}\right)^{1 / 2} \bar{P} \\
* & -\bar{M}_{1} & 0 & 0 & 0 & 0 \\
* & * & -\bar{M}_{2} & 0 & 0 & 0 \\
* & * & * & -\bar{M}_{3} & 0 & 0 \\
* & * & * & * & -I_{n} & 0 \\
* & * & * & * & * & -\bar{W}
\end{array}\right]
$$

$$
\begin{gathered}
\frac{m}{1-\sigma_{2}}-2 \alpha p \leq 0, \quad P \geq p I_{n}, \quad \bar{P} \geq \bar{p} I_{n}, \\
\frac{l}{1-\tau_{2}}-2 \beta \bar{p} \leq 0, \\
L_{2} M_{2} L_{2}-(1-\tau) Q \leq 0, \quad M_{3}-\left(1-\tau_{1}\right) U \leq 0, \\
\bar{L}_{2} \bar{M}_{2} \bar{L}_{2}-(1-\sigma) \bar{Q} \leq 0, \quad \bar{M}_{3}-\left(1-\sigma_{1}\right) \bar{U} \leq 0,
\end{gathered}
$$

and the adaptive feedback controllers are designed as

$$
\begin{gathered}
u_{i}(t)=-\left[\gamma_{i}+\alpha_{i}(t)\right] e_{i}(t), \\
v_{j}(t)=-\left[\eta_{j}+\beta_{j}(t)\right] \varepsilon_{j}(t), \\
\alpha_{i}(t)= \begin{cases}\frac{\left\|\bar{G}\left(\dot{e}_{i}(t)\right)\right\|^{2}}{\left\|e_{i}(t)\right\|^{2}} \alpha, & \left\|e_{i}(t)\right\|^{2} \neq 0, \\
0, & \left\|e_{i}(t)\right\|^{2}=0,\end{cases} \\
\beta_{j}(t)= \begin{cases}\frac{\left\|G\left(\dot{\varepsilon}_{j}(t)\right)\right\|^{2}}{\left\|\varepsilon_{j}(t)\right\|^{2}} \beta, & \left\|\varepsilon_{j}(t)\right\|^{2} \neq 0, \\
0, & \left\|\varepsilon_{j}(t)\right\|^{2}=0,\end{cases}
\end{gathered}
$$

where $Z_{r i}=-2 P D_{r}+\bar{L}_{3} \bar{H} \bar{W} \bar{H} \bar{L}_{3}+L_{1} M_{1} L_{1}+m \bar{U}-2 \gamma_{i} P+Q$, $\bar{Z}_{r j}=-2 \bar{P} \bar{D}_{r}+L_{3} H W H L_{3}+\bar{L}_{1} \bar{M}_{1} \bar{L}_{1}+l U+\bar{Q}-2 \eta_{j} \bar{P}, r \in \aleph$, $i=1,2, \ldots, l$, and $j=1,2, \ldots, m$.

Proof. For the error dynamical system (6), we design the following Lyapunov-Krasovskii function:

$$
V(t)=V_{1}(E(t))+V_{2}(E(t)),
$$

where $E(t)=\left(e_{1}^{T}(t), e_{2}^{T}(t), \ldots, e_{l}^{T}(t), \varepsilon_{1}^{T}(t), \varepsilon_{2}^{T}(t), \ldots, \varepsilon_{m}^{T}(t)\right)^{T}$,

$$
\begin{aligned}
V_{1}= & \sum_{i=1}^{l} e_{i}^{T}(t) P e_{i}(t)+\sum_{i=1}^{l} \int_{t-\tau(t)}^{t} e_{i}^{T}(s) Q e_{i}(s) \mathrm{d} s \\
& +\sum_{j=1}^{m} \sum_{i=1}^{n} w_{i} \int_{0}^{\infty} h_{i}(v) \mathrm{d} v \int_{0}^{\infty} h_{i}(\theta) \\
& \times \int_{t-\theta}^{t} K_{i}^{2}\left(\varepsilon_{j i}(s)\right) \mathrm{d} s \mathrm{~d} \theta \\
& +l \sum_{j=1}^{m} \int_{t-\tau_{1}(t)}^{t} \varepsilon_{j}^{T}(s) U \varepsilon_{j}(s) \mathrm{d} s \\
& +\frac{l}{1-\tau_{2}} \sum_{j=1}^{m} \int_{t-\tau_{2}(t)}^{t} G^{T}\left(\dot{\varepsilon}_{j}(s)\right) G\left(\dot{\varepsilon}_{j}(s)\right) \mathrm{d} s,
\end{aligned}
$$




$$
\begin{aligned}
V_{2}= & \sum_{j=1}^{m} \varepsilon_{j}^{T}(t) \bar{P} \varepsilon_{j}(t)+\sum_{j=1}^{m} \int_{t-\mu(t)}^{t} \varepsilon_{j}^{T}(s) \bar{Q} \varepsilon_{j}(s) \mathrm{d} s \\
& +\sum_{i=1}^{l} \sum_{j=1}^{n} \bar{w}_{j} \int_{0}^{\infty} \bar{h}_{j}(v) \mathrm{d} v \int_{0}^{\infty} \bar{h}_{j}(\theta) \\
& \times \int_{t-\theta}^{t} \bar{K}_{j}\left(e_{i j}(s)\right) \mathrm{d} s \mathrm{~d} \theta \\
& +m \sum_{i=1}^{l} \int_{t-\mu_{1}(t)}^{t} e_{i}^{T}(s) \bar{U} e_{i}(s) \mathrm{d} s \\
& +\frac{m}{1-\sigma_{2}} \sum_{i=1}^{l} \int_{t-\mu_{2}(t)}^{t} \bar{G}^{T}\left(\dot{e}_{i}(s)\right) \bar{G}\left(\dot{e}_{i}(s)\right) \mathrm{d} s .
\end{aligned}
$$

$$
\begin{aligned}
& +l \sum_{j=1}^{m} \varepsilon_{j}^{T}(s) U \varepsilon_{j}(s)-\left(1-\dot{\tau}_{1}(t)\right) l \\
& \times \sum_{j=1}^{m} \varepsilon_{j}^{T}\left(t-\tau_{1}(t)\right) U \varepsilon_{j}\left(t-\tau_{1}(t)\right) \\
& +\frac{l}{1-\tau_{2}} \sum_{j=1}^{m} G^{T}\left(\dot{\varepsilon}_{j}(t)\right) G\left(\dot{\varepsilon}_{j}(t)\right) \\
& -\frac{1-\dot{\tau}_{2}(t)}{1-\tau_{2}} l \\
& \left.\times \sum_{j=1}^{m} G^{T}\left(\dot{\varepsilon}_{j}\left(t-\tau_{2}(t)\right)\right) G\left(\dot{\varepsilon}_{j}\left(t-\tau_{2}(t)\right)\right)\right\} .
\end{aligned}
$$

By Lemma 1, we can get from $\left(S_{1}\right)$

Calculating the derivative of (22) along the trajectories of (6), we have

$$
\begin{aligned}
& \dot{V}_{1}=\sum_{r=1}^{N} \xi_{r}(t, \lambda) \\
& \times\left\{\sum_{i=1}^{l} 2 e_{i}^{T}(t) P\right. \\
& \times\left[-D_{r} e_{i}(t)+R_{r 1} F_{1}\left(e_{i}(t)\right)\right. \\
& +R_{r 2} F_{2}\left(e_{i}(t-\tau(t))\right) \\
& +\sum_{j=1}^{m} a_{r i j} \varepsilon_{j}\left(t-\tau_{1}(t)\right) \\
& +\sum_{j=1}^{m} b_{r i j} G\left(\dot{\varepsilon}_{j}\left(t-\tau_{2}(t)\right)\right)+\sum_{j=1}^{m} c_{r i j} \\
& \left.\times \int_{-\infty}^{t} h(t-s) K\left(\varepsilon_{j}(s)\right) d s+u_{i}(t)\right] \\
& +\sum_{i=1}^{l} e_{i}^{T}(t) Q e_{i}(t)-(1-\dot{\tau}(t)) \\
& \times \sum_{i=1}^{l} e_{i}^{T}(t-\tau(t)) Q e_{i}(t-\tau(t)) \\
& +\sum_{j=1}^{m} \sum_{i=1}^{n} w_{i}\left(K_{i}\left(\varepsilon_{j i}(t)\right) \int_{0}^{\infty} h_{i}(v) d v\right)^{2} \\
& -\sum_{j=1}^{m} \sum_{i=1}^{n} w_{i} \int_{0}^{\infty} h_{i}(v) d v \\
& \times \int_{0}^{\infty} h_{i}(\theta) K_{i}^{2}\left(\varepsilon_{j i}(t-\theta)\right) d \theta
\end{aligned}
$$

$$
\begin{aligned}
& 2 e_{i}^{T}(t) P R_{r 1} F_{1}\left(e_{i}(t)\right) \\
& \leq e_{i}^{T}(t) P R_{r 1} M_{1}^{-1} R_{r 1}^{T} P e_{i}(t) \\
&+F_{1}^{T}\left(e_{i}(t)\right) M_{1} F_{1}\left(e_{i}(t)\right) \\
& \leq e_{i}^{T}(t)\left(P R_{r 1} M_{1}^{-1} R_{r 1}^{T} P+L_{1} M_{1} L_{1}\right) e_{i}(t), \\
& 2 e_{i}^{T}(t) P R_{r 2} F_{2}\left(e_{i}(t-\tau(t))\right) \\
& \leq e_{i}^{T}(t) P R_{r 2} M_{2}^{-1} R_{r 2}^{T} P e_{i}(t) \\
& \quad+F_{2}^{T}\left(e_{i}(t-\tau(t))\right) M_{2} F_{2}\left(e_{i}(t-\tau(t))\right) \\
& \leq e_{i}^{T}(t) P R_{r 2} M_{2}^{-1} R_{r 2}^{T} P e_{i}(t) \\
& \quad+e_{i}^{T}(t-\tau(t)) L_{2} M_{2} L_{2} e_{i}(t-\tau(t)), \\
& 2 a_{r i j} e_{i}^{T}(t) P \varepsilon_{j}\left(t-\tau_{1}(t)\right) \\
& \leq a_{r i j}^{2} e_{i}^{T}(t) P M_{3}^{-1} P e_{i}(t) \\
&+\varepsilon_{j}^{T}\left(t-\tau_{1}(t)\right) M_{3} \varepsilon_{j}^{T}\left(t-\tau_{1}(t)\right) .
\end{aligned}
$$

By assumptions $\left(S_{1}\right)$ and $\left(S_{4}\right)$, it is obvious that

$$
\begin{gathered}
\sum_{j=1}^{m} \sum_{i=1}^{n} w_{i}\left(K_{i}\left(\varepsilon_{j i}(t)\right) \int_{0}^{\infty} h_{i}(v) \mathrm{d} v\right)^{2} \\
=\sum_{j=1}^{m} K^{T}\left(\varepsilon_{j}(t)\right) H W H K\left(\varepsilon_{j}(t)\right) \\
\leq \sum_{j=1}^{m} \varepsilon_{j}^{T}(t) L_{3} H W H L_{3} \varepsilon_{j}(t), \\
2 \sum_{i=1}^{l} c_{r i j} e_{i}^{T}(t) P \int_{-\infty}^{t} h(t-s) K\left(\varepsilon_{j}(s)\right) \mathrm{d} s \\
\leq l \sum_{i=1}^{l} c_{r i j}^{2} e_{i}^{T}(t) P W^{-1} P e_{i}(t) \\
+\frac{1}{l} \sum_{i=1}^{l}\left(\int_{-\infty}^{t} h(t-s) K\left(\varepsilon_{j}(s)\right) \mathrm{d} s\right)^{T}
\end{gathered}
$$




$$
\begin{aligned}
& \times W \int_{-\infty}^{t} h(t-s) K\left(\varepsilon_{j}(s)\right) \mathrm{d} s \\
= & l \sum_{i=1}^{l} c_{r i j}^{2} e_{i}^{T}(t) P W^{-1} P e_{i}(t) \\
& +\left(\int_{-\infty}^{t} h(t-s) K\left(\varepsilon_{j}(s)\right) \mathrm{d} s\right)^{T} \\
& \times W \int_{-\infty}^{t} h(t-s) K\left(\varepsilon_{j}(s)\right) \mathrm{d} s .
\end{aligned}
$$

Observe that

$$
\begin{aligned}
& -\frac{l\left(1-\dot{\tau}_{2}(t)\right)}{1-\tau_{2}} \sum_{j=1}^{m} G^{T}\left(\dot{\varepsilon}_{j}\left(t-\tau_{2}(t)\right)\right) G\left(\dot{\varepsilon}_{j}\left(t-\tau_{2}(t)\right)\right) \\
& \quad+2 \sum_{i=1}^{l} \sum_{j=1}^{m} e_{i}^{T}(t) P b_{r i j} G\left(\dot{\varepsilon}_{j}\left(t-\tau_{2}(t)\right)\right) \\
& \leq-\sum_{i=1}^{l} \sum_{j=1}^{m} G^{T}\left(\dot{\varepsilon}_{j}\left(t-\tau_{2}(t)\right)\right) G\left(\dot{\varepsilon}_{j}\left(t-\tau_{2}(t)\right)\right) \\
& \quad+2 \sum_{i=1}^{l} \sum_{j=1}^{m} e_{i}^{T}(t) P b_{r i j} G\left(\dot{\varepsilon}_{j}\left(t-\tau_{2}(t)\right)\right) \\
& =-\sum_{i=1}^{l} \sum_{j=1}^{m}\left(b_{r i j} P e_{i}(t)-G\left(\dot{\varepsilon}_{j}\left(t-\tau_{2}(t)\right)\right)\right)^{T} \\
& \quad+\sum_{i=1}^{l} \sum_{j=1}^{m} b_{r i j}^{2} e_{i}^{T}(t) P^{2} e_{i}(t) \\
& +\sum_{j=1}^{m} b_{r i j}^{2} e_{i}^{T}(t) P^{2} e_{i}(t) . \\
& \times\left(b_{r i j} P e_{i}(t)-G\left(\dot{\varepsilon}_{j}\left(t-\tau_{2}(t)\right)\right)\right)
\end{aligned}
$$

Using inequality

$$
\int_{0}^{\infty} f^{2}(s) \mathrm{d} s \int_{0}^{\infty} g^{2}(s) \mathrm{d} s \geq\left(\int_{0}^{\infty} f(s) g(s) \mathrm{d} s\right)^{2},
$$

we have

$$
\begin{aligned}
\sum_{i=1}^{n} w_{i} \int_{0}^{\infty} h_{i}(v) \mathrm{d} v \int_{0}^{\infty} h_{i}(\theta) K_{i}^{2}\left(\varepsilon_{j i}(t-\theta)\right) \mathrm{d} \theta \\
\geq \sum_{i=1}^{n} w_{i}\left(\int_{0}^{\infty} h_{i}(\theta) K_{i}\left(\varepsilon_{j i}(t-\theta)\right) \mathrm{d} \theta\right)^{2} \\
=\left(\int_{-\infty}^{t} h(t-s) K\left(\varepsilon_{j}(s)\right) \mathrm{d} s\right)^{T} \\
\quad \times W \int_{-\infty}^{t} h(t-s) K\left(\varepsilon_{j}(s)\right) \mathrm{d} s
\end{aligned}
$$

Using Lemma 2 and condition (17), we get

$$
-e^{T}(t) P \frac{\left\|\bar{G}\left(\dot{e}_{i}(t)\right)\right\|^{2}}{\left\|e_{i}(t)\right\|^{2}} e(t) \leq-p \bar{G}^{T}\left(\dot{e}_{i}(t)\right) \bar{G}\left(\dot{e}_{i}(t)\right) .
$$

Substituting (20) into (24) and combining (24)-(31), it can be derived by condition (18) that

$$
\dot{V}_{1} \leq \sum_{r=1}^{N} \xi_{r}(t, \lambda)
$$

$$
\begin{aligned}
& \times\left\{\sum_{i=1}^{l} e_{i}^{T}(t) \Omega_{r i} e_{i}(t)\right. \\
& +\sum_{i=1}^{l} e_{i}^{T}(t-\tau(t))\left[L_{2} M_{2} L_{2}-(1-\tau) Q\right] \\
& \quad \times e_{i}(t-\tau(t)) \\
& +\sum_{j=1}^{m} \varepsilon_{j}^{T}(s)\left(l U+L_{3} H W H L_{3}\right) \varepsilon_{j}(s) \\
& +\sum_{j=1}^{m} \varepsilon_{j}^{T}\left(t-\tau_{1}(t)\right)\left[l M_{3}-l\left(1-\tau_{1}\right) U\right] \\
& \quad \times \frac{l}{1-\tau_{2}} \sum_{j=1}^{m} G^{T}\left(t-\dot{\varepsilon}_{j}(t)\right) G\left(\dot{\varepsilon}_{j}(t)\right) \\
& \left.+2 \alpha p \sum_{i=1}^{l} \bar{G}^{T}\left(\dot{e}_{i}(t)\right) \bar{G}\left(\dot{e}_{i}(t)\right)\right\}
\end{aligned}
$$$$
\leq \sum_{r=1}^{N} \xi_{r}(t, \lambda)
$$

$$
\times\left\{\sum_{i=1}^{l} e_{i}^{T}(t) \Omega_{r i} e_{i}(t)\right.
$$$$
+\sum_{j=1}^{m} \varepsilon_{j}^{T}(t)\left(l U+L_{3} H W H L_{3}\right) \varepsilon_{j}(t)
$$$$
+\frac{l}{1-\tau_{2}} \sum_{j=1}^{m} G^{T}\left(\dot{\varepsilon}_{j}(t)\right) G\left(\dot{\varepsilon}_{j}(t)\right)
$$$$
\left.-2 \alpha p \sum_{i=1}^{l} \bar{G}^{T}\left(\dot{e}_{i}(t)\right) \bar{G}\left(\dot{e}_{i}(t)\right)\right\} \text {, }
$$

where $\Omega_{i}=-2 P D_{r}+P R_{r 1} M_{1}^{-1} R_{r 1}^{T} P+L_{1} M_{1} L_{1}+$ $P R_{r 2} M_{2}^{-1} R_{r 2}^{T} P+\sum_{j=1}^{m} a_{r i j}^{2} P M_{3}^{-1} P+\sum_{j=1}^{m} b_{r i j}^{2} P^{2}+$ $l \sum_{j=1}^{m} c_{r i j}^{2} P W^{-1} P-2 \gamma_{i} P+Q$. 
Meanwhile, by a similar process, the following inequality can be true:

$$
\begin{aligned}
\dot{V}_{2} \leq \sum_{r=1}^{N} \xi_{r}(t, \lambda) & \\
\times & \left\{\sum_{j=1}^{m} \varepsilon_{j}^{T}(t) \bar{\Omega}_{r j} \varepsilon_{j}(t)\right. \\
& +\sum_{i=1}^{l} e_{i}^{T}(t)\left(m \bar{U}+\bar{L}_{3} \bar{H} \bar{W} \bar{H} \bar{L}_{3}\right) e_{i}(t) \\
& +\frac{m}{1-\sigma_{2}} \sum_{i=1}^{l} \bar{G}^{T}\left(\dot{e}_{i}(t)\right) \bar{G}\left(\dot{e}_{i}(t)\right) \\
& \left.-2 \beta \bar{p} \sum_{j=1}^{m} G^{T}\left(\dot{\varepsilon}_{j}(t)\right) G\left(\dot{\varepsilon}_{j}(t)\right)\right\}
\end{aligned}
$$

where $\bar{\Omega}_{j}=-2 \bar{P} \bar{D}_{r}+\bar{P} \bar{R}_{r 1} \bar{M}_{1}^{-1} \bar{R}_{r 1}^{T} \bar{P}+\bar{L}_{1} \bar{M}_{1} \bar{L}_{1}+$ $\bar{P} \bar{R}_{r 2} \bar{M}_{2}^{-1} \bar{R}_{r 2}^{T} \bar{P}+\sum_{i=1}^{l} \bar{a}_{r i j}^{2} \bar{P} \bar{M}_{3}^{-1} \bar{P}+\sum_{i=1}^{l} \bar{b}_{r j i}^{2} \bar{P}^{2}+$ $m \sum_{i=1}^{l} \bar{c}_{r j i}^{2} \bar{P} \bar{W}^{-1} \bar{P}-2 \eta_{j} \bar{P}+\bar{Q}$.

By condition (17), we have

$$
\begin{aligned}
\dot{V} \leq \sum_{r=1}^{N} \xi_{r}( & t, \lambda) \\
& \times\left[\sum_{i=1}^{l} e_{i}^{T}(t)\left(\Omega_{r i}+m \bar{U}+\bar{L}_{3} \bar{H} \bar{W} \bar{H} \bar{L}_{3}\right) e_{i}(t)\right. \\
& \left.+\sum_{j=1}^{m} \varepsilon_{j}^{T}(t)\left(\bar{\Omega}_{r j}+l U+L_{3} H W H L_{3}\right) \varepsilon_{j}(t)\right] .
\end{aligned}
$$

By (15)-(16) and Lemma 3 (Schur complement), it can be obtained that $\Omega_{r i}+m \bar{U}+\bar{L}_{3} \bar{H} \bar{W} \bar{H} \bar{L}_{3}<0, \bar{\Omega}_{r j}+l U+$ $L_{3} H W H L_{3}<0$. Set $\rho=\min \left\{\rho_{1}, \rho_{2}\right\}$, where

$$
\begin{gathered}
\rho_{1}=-\min \left\{\rho_{\min }\left(\Omega_{r i}+m \bar{U}+\bar{L}_{3} \bar{H} \bar{W} \bar{H} \bar{L}_{3}\right),\right. \\
r \in \aleph, 1 \leq i \leq l\}, \\
\rho_{2}=-\min \left\{\rho_{\min }\left(\bar{\Omega}_{r j}+l U+L_{3} H W H L_{3}\right),\right. \\
r \in \aleph, 1 \leq j \leq m\},
\end{gathered}
$$

then $\rho>0$, and

$$
\begin{aligned}
\dot{V} & \leq-\rho_{1} \sum_{i=1}^{l} e_{i}^{T}(t) e_{i}(t)-\rho_{2} \sum_{j=1}^{m} \varepsilon_{j}^{T}(t) \varepsilon_{j}(t) \\
& \leq-\rho E^{T}(t) E(t) .
\end{aligned}
$$

Therefore, $V$ is nonincreasing in $t \geq 0$. One has $V$ bounded since $0 \leq V(t, E(t)) \leq V(0, E(0))$, so $\lim _{t \rightarrow+\infty} V(t, E(t))$ exists and

$$
\begin{aligned}
\lim _{t \rightarrow+\infty} \int_{0}^{t} E^{T}(s) E(s) \mathrm{d} s \\
\quad \leq-\frac{1}{\rho} \lim _{t \rightarrow+\infty} \int_{0}^{t} \frac{\mathrm{d} V}{\mathrm{~d} s} \mathrm{~d} s \\
\quad=\frac{1}{\rho} V(0, E(0))-\frac{1}{\rho} \lim _{t \rightarrow+\infty} V(t, E(t)) .
\end{aligned}
$$

From (22)-(23) and conditions $P \geq p I, \bar{P} \geq \bar{p} I$ and we have $0 \leq E^{T}(t) E(t) \leq \max \{1 / p, 1 / \bar{p}\} V(t, E(t))$, so $E^{T}(t) E(t)$ is bounded. According to error system (6), $(\mathrm{d} / \mathrm{d} t) E^{T}(t) E(t)=$ $2 E^{T}(t) \dot{E}(t)$ is bounded for $t \geq 0$ due to the boundedness of activation functions. From the above we can see that $E(t) \epsilon$ $L^{2} \cap L^{\infty}$ and $(\mathrm{d} / \mathrm{d} t) E^{T}(t) E(t) \in L^{\infty}$. By using Barbálat lemma (see [36]), one has $\lim _{t \rightarrow+\infty} E^{T}(t) E(t)=0$, so the two SBNNs (4) and (5) can obtain synchronization under the controllers (20). This completes the proof.

We take CBDN (1) as drive network. The response network of the drive network (1) is

$$
\begin{aligned}
& \dot{\hat{x}}_{i}(t)=-D \widehat{x}_{i}(t)+R_{1} f_{1}\left(\widehat{x}_{i}(t)\right)+R_{2} f_{2}\left(\widehat{x}_{i}(t-\tau(t))\right) \\
& +I(t)+\sum_{j=1}^{m} a_{i j} \widehat{y}_{j}\left(t-\tau_{1}(t)\right) \\
& +\sum_{j=1}^{m} b_{i j} g\left(\dot{\hat{y}}_{j}\left(t-\tau_{2}(t)\right)\right) \\
& +\sum_{j=1}^{m} c_{i j} \int_{-\infty}^{t} h(t-s) k\left(\widehat{y}_{j}(s)\right) \mathrm{d} s+u_{i}(t), \\
& \dot{\hat{y}}_{j}(t)=-\bar{D} \widehat{y}_{j}(t)+\bar{R}_{1} \bar{f}_{1}\left(\widehat{y}_{j}(t)\right)+\bar{R}_{2} \bar{f}_{2}\left(\widehat{y}_{j}(t-\sigma(t))\right) \\
& +J(t)+\sum_{i=1}^{l} \bar{a}_{j i} \widehat{x}_{i}\left(t-\sigma_{1}(t)\right) \\
& +\sum_{i=1}^{l} \bar{b}_{j i} \overline{\mathcal{g}}\left(\dot{\hat{x}}_{i}\left(t-\sigma_{2}(t)\right)\right) \\
& +\sum_{i=1}^{l} \bar{c}_{j i} \int_{-\infty}^{t} \bar{h}(t-s) \bar{k}\left(\widehat{x}_{i}(s)\right) \mathrm{d} s+v_{j}(t),
\end{aligned}
$$

where $u_{i}(t), v_{j}(t) \in R^{n}$ are the control inputs.

From Theorem 4, we can get the following corollary. 
Corollary 5. Under assumptions $\left(S_{1}\right)-\left(S_{4}\right)$, the two coupled CBDNs (1) and (38) can be synchronized, if there exist positive constants $\alpha, \beta, p, \bar{p}, \gamma_{i}, \eta_{j}(i=1,2, \ldots, l, j=1,2, \ldots, m)$, $n \times n$ positive matrices $P, Q, U, \bar{P}, \bar{Q}, \bar{U}$ and $n \times n$ diagonal positive matrices $W=\operatorname{diag}\left(w_{1}, w_{2}, \ldots, w_{n}\right), \bar{W}=$ $\operatorname{diag}\left(\bar{w}_{1}, \bar{w}_{2}, \ldots, \bar{w}_{n}\right), M_{i}, \bar{M}_{i}(i=1,2,3)$ such that

$$
\left[\begin{array}{cccccc}
Z_{i} & P R_{1} & P R_{2} & \left(\sum_{j=1}^{m} a_{i j}^{2}\right)^{1 / 2} P & \left(\sum_{j=1}^{m} b_{i j}^{2}\right)^{1 / 2} P & \left(l \sum_{j=1}^{m} c_{i j}^{2}\right)^{1 / 2} P \\
* & -M_{1} & 0 & 0 & 0 & 0 \\
* & * & -M_{2} & 0 & 0 & 0 \\
* & * & * & -M_{3} & 0 & 0 \\
* & * & * & * & -I_{n} & 0 \\
* & * & * & * & * & -W
\end{array}\right]
$$

$<0$,

$$
\begin{aligned}
& {\left[\begin{array}{cccccc}
\bar{Z}_{j} & \bar{P} \bar{R}_{1} & \bar{P} \bar{R}_{2} & \left(\sum_{i=1}^{l} \bar{a}_{j i}^{2}\right)^{1 / 2} \bar{P} & \left(\sum_{i=1}^{l} \bar{b}_{j i}^{2}\right)^{1 / 2} \bar{P} & \left(m \sum_{i=1}^{l} \bar{c}_{j i}^{2}\right)^{1 / 2} \bar{P} \\
* & -\bar{M}_{1} & 0 & 0 & 0 & 0 \\
* & * & -\bar{M}_{2} & 0 & 0 & 0 \\
* & * & * & -\bar{M}_{3} & 0 & 0 \\
* & * & * & * & -I_{n} & 0 \\
* & * & * & * & * & -\bar{W}
\end{array}\right]} \\
& <0 \\
& \frac{m}{1-\sigma_{2}}-2 \alpha p \leq 0, \quad P \geq p I_{n}, \quad \bar{P} \geq \bar{p} I_{n}, \\
& \frac{l}{1-\tau_{2}}-2 \beta \bar{p} \leq 0, \\
& L_{2} M_{2} L_{2}-(1-\tau) Q \leq 0, \quad M_{3}-\left(1-\tau_{1}\right) U \leq 0, \\
& \bar{L}_{2} \bar{M}_{2} \bar{L}_{2}-(1-\sigma) \bar{Q} \leq 0, \quad \bar{M}_{3}-\left(1-\sigma_{1}\right) \bar{U} \leq 0 \text {, }
\end{aligned}
$$

and the adaptive feedback controllers are designed as

$$
\begin{gathered}
u_{i}(t)=-\left[\gamma_{i}+\alpha_{i}(t)\right] e_{i}(t), \\
v_{j}(t)=-\left[\eta_{j}+\beta_{j}(t)\right] \varepsilon_{j}(t), \\
\alpha_{i}(t)= \begin{cases}\frac{\left\|\bar{G}\left(\dot{e}_{i}(t)\right)\right\|^{2}}{\left\|e_{i}(t)\right\|^{2}} \alpha, & \left\|e_{i}(t)\right\|^{2} \neq 0, \\
0, & \left\|e_{i}(t)\right\|^{2}=0,\end{cases} \\
\beta_{j}(t)= \begin{cases}\frac{\left\|G\left(\dot{\varepsilon}_{j}(t)\right)\right\|^{2}}{\left\|\varepsilon_{j}(t)\right\|^{2}} \beta, & \left\|\varepsilon_{j}(t)\right\|^{2} \neq 0, \\
0, & \left\|\varepsilon_{j}(t)\right\|^{2}=0,\end{cases}
\end{gathered}
$$

where $Z_{i}=-2 P D+\bar{L}_{3} \bar{H} \bar{W} \bar{H} \bar{L}_{3}+L_{1} M_{1} L_{1}+m \bar{U}-2 \gamma_{i} P+$ $Q, \bar{Z}_{j}=-2 \bar{P} \bar{D}+L_{3} H W H L_{3}+\bar{L}_{1} \bar{M}_{1} \bar{L}_{1}+l U-2 \eta_{j} \bar{P}+\bar{Q}, i=$ $1,2, \ldots, l, j=1,2, \ldots, m$.
Remark 6. From Corollary 5, we can easily get that the controllers in this paper are simpler than those of Theorem 1 in [29].

Remark 7. If the coupling matrix of the SCBNN is not a diffusive matrix satisfying the sum of every row being zero, we can still obtain the same result from the proof of Theorem 4.

Theorem 8 presents another sufficient condition to ascertain that the two networks (4) and (5) can be synchronized, using the following simple adaptive feedback controllers:

$$
\begin{aligned}
& u_{i}(t)=-\gamma_{i} e_{i}(t), \\
& v_{j}(t)=-\bar{\gamma}_{j} \varepsilon_{j}(t),
\end{aligned}
$$

where $i=1,2, \ldots, l, j=1,2, \ldots, m, \gamma_{i}$, and $\bar{\gamma}_{j}$ are positive constants.

Let

$$
\begin{gathered}
e(t)=\left(e_{1}^{T}(t), e_{2}^{T}(t), \ldots, e_{l}^{T}(t)\right)^{T}, \\
\varepsilon(t)=\left(\varepsilon_{1}^{T}(t), \varepsilon_{2}^{T}(t), \ldots, \varepsilon_{m}^{T}(t)\right)^{T}, \\
\widetilde{F}_{k}(e(t))=\left(F_{k}^{T}\left(e_{1}(t)\right), F_{k}^{T}\left(e_{2}(t)\right), \ldots, F_{k}^{T}\left(e_{l}(t)\right)\right)^{T}, \\
\widehat{F}_{k}(e(t))=\left(\bar{F}_{k}^{T}\left(\varepsilon_{1}(t)\right), \bar{F}_{k}^{T}\left(\varepsilon_{2}(t)\right), \ldots, \bar{F}_{k}^{T}\left(\varepsilon_{m}(t)\right)\right)^{T}, \\
\widetilde{G}(\dot{\varepsilon}(t))=\left(G^{T}\left(\dot{\varepsilon}_{1}(t)\right), G^{T}\left(\dot{\varepsilon}_{2}(t)\right), \ldots, G^{T}\left(\dot{\varepsilon}_{m}(t)\right)\right)^{T}, \\
\widehat{G}(\dot{e}(t))=\left(\bar{G}^{T}\left(\dot{e}_{1}(t)\right), \bar{G}^{T}\left(\dot{e}_{2}(t)\right), \ldots, \bar{G}^{T}\left(\dot{e}_{l}(t)\right)\right)^{T}, \\
\widetilde{K}(\varepsilon(t))=\left(K^{T}\left(\varepsilon_{1}(t)\right), K^{T}\left(\varepsilon_{2}(t)\right), \ldots, K^{T}\left(\varepsilon_{m}(t)\right)\right)^{T}, \\
\widehat{K}(e(t))=\left(\bar{K}^{T}\left(e_{1}(t)\right), \bar{K}^{T}\left(e_{2}(t)\right), \ldots, \bar{K}^{T}\left(e_{l}(t)\right)\right)^{T}, \\
\widetilde{\Gamma}^{T}=-\operatorname{diag}\left(\gamma_{1}, \gamma_{2}, \ldots, \gamma_{l}\right),
\end{gathered}
$$

then the error dynamical system of (6) becomes

$$
\begin{aligned}
\dot{e}(t)=\sum_{r=1}^{N} \xi_{r}(t, \lambda) & \\
& \quad \times\left[-\left(I_{l} \otimes D_{r}\right) e(t)+\left(I_{l} \otimes R_{r 1}\right) \widetilde{F}_{1}(e(t))\right. \\
& \quad+\left(I_{l} \otimes R_{r 2}\right) \widetilde{F}_{2}(e(t-\tau(t)))
\end{aligned}
$$




$$
\begin{aligned}
& +\left(A_{r} \otimes I_{n}\right) \varepsilon\left(t-\tau_{1}(t)\right) \\
& +\left(B_{r} \otimes I_{n}\right) \widetilde{G}\left(\dot{\varepsilon}\left(t-\tau_{2}(t)\right)\right) \\
& +\left(C_{r} \otimes I_{n}\right) \int_{-\infty}^{t}\left(I_{m} \otimes h(t-s)\right) \widetilde{K}(\varepsilon(s)) d s \\
& \left.+\left(\widetilde{\Gamma} \otimes I_{n}\right) e(t)\right] \\
\dot{\varepsilon}(t)=\sum_{r=1}^{N} \xi_{r}(t, \lambda) & \\
\times[- & \left(I_{m} \otimes \bar{D}\right) \varepsilon(t)+\left(I_{m} \otimes \bar{R}_{r 1}\right) \widehat{F}_{1}(\varepsilon(t)) \\
& +\left(I_{m} \otimes \bar{R}_{r 2}\right) \widehat{F}_{2}(\varepsilon(t-\sigma(t))) \\
& +\left(\bar{A}_{r} \otimes I_{n}\right) e\left(t-\sigma_{1}(t)\right)
\end{aligned}
$$$$
+\left(\bar{B}_{r} \otimes I_{n}\right) \widehat{G}\left(\dot{e}\left(t-\sigma_{2}(t)\right)\right)
$$$$
+\left(\bar{C}_{r} \otimes I_{n}\right) \int_{-\infty}^{t}\left(I_{l} \otimes h(t-s)\right) \widehat{K}(e(s)) d s
$$$$
\left.+\left(\widehat{\Gamma} \otimes I_{n}\right) \varepsilon(t)\right]
$$

Theorem 8. Under assumptions $\left(S_{1}\right)-\left(S_{4}\right)$ and using the adaptive feedback controllers (41), the two coupled SCBNNs (4) and (5) can be synchronized, if there exist $n \times n$ positive matrices $P, U, \bar{P}, \bar{U}$ and $n \times n$ diagonal positive matrices $W=$ $\operatorname{diag}\left(w_{1}, w_{2}, \ldots, w_{n}\right), \bar{W}=\operatorname{diag}\left(\bar{w}_{1}, \bar{w}_{2}, \ldots, \bar{w}_{n}\right), Q, V, M, \bar{Q}$, $\bar{V}, \bar{M}$ such that for $r \in \aleph$, the following matrix inequalities hold:

$$
\begin{aligned}
& \Omega_{r}=\left(\begin{array}{cccccc}
\Psi_{r 1} & \Psi_{r 2} & \Psi_{r 3} & \Psi_{r 4} & \Psi_{r 5} & \Psi_{r 6} \\
* & \Psi_{r 7} & I_{l} \otimes\left(R_{r 1}^{T} \bar{V} R_{r 2}\right) & A_{r} \otimes\left(R_{r 1}^{T} \bar{V}\right) & B_{r} \otimes\left(R_{r 1}^{T} \bar{V}\right) & C_{r} \otimes\left(R_{r 1}^{T} \bar{V}\right) \\
* & * & \Psi_{r 8} & A_{r} \otimes\left(R_{r 2}^{T} \bar{V}\right) & B_{r} \otimes\left(R_{r 2}^{T} \bar{V}\right) & C_{r} \otimes\left(R_{r 2}^{T} \bar{V}\right) \\
* & * & * & \Psi_{r 9} & \left(A_{r}^{T} B_{r}\right) \otimes \bar{V} & \left(A_{r}^{T} C_{r}\right) \otimes \bar{V} \\
* & * & * & * & \Psi_{r 10} & \left(B_{r}^{T} C_{r}\right) \otimes \bar{V} \\
* & * & * & * & * & \Psi_{r 11}
\end{array}\right)<0, \\
& \bar{\Omega}_{r}=\left(\begin{array}{cccccc}
\bar{\Psi}_{r 1} & \bar{\Psi}_{r 2} & \bar{\Psi}_{r 3} & \bar{\Psi}_{r 4} & \bar{\Psi}_{r 5} & \bar{\Psi}_{r 6} \\
* & \bar{\Psi}_{r 7} & I_{l} \otimes\left(\bar{R}_{r 1}^{T} V \bar{R}_{r 2}\right) & \bar{A}_{r} \otimes\left(\bar{R}_{r 1}^{T} V\right) & \bar{B}_{r} \otimes\left(\bar{R}_{r 1}^{T} V\right) & \bar{C}_{r} \otimes\left(\bar{R}_{r 1}^{T} V\right) \\
* & * & \bar{\Psi}_{r 8} & \bar{A}_{r} \otimes\left(\bar{R}_{r 2}^{T} V\right) & \bar{B}_{r} \otimes\left(\bar{R}_{r 2}^{T} V\right) & \bar{C}_{r} \otimes\left(\bar{R}_{r 2}^{T} V\right) \\
* & * & * & \bar{\Psi}_{r 9} & \left(\bar{B}_{r}\right) \otimes V & \left(\bar{A}_{r}^{T} \bar{C}_{r}\right) \otimes V \\
* & * & * & * & \bar{\Psi}_{r 10} & \bar{B}_{r}^{T} \bar{C}_{r} \otimes V \\
* & * & * & * & * & \bar{\Psi}_{r 11}
\end{array}\right)<0,
\end{aligned}
$$

with

$$
\begin{aligned}
\Psi_{r 1}= & I_{l} \otimes\left(-P D_{r}-D_{r}^{T} P+Q+\bar{U}\right. \\
& \left.+\bar{L}_{3} \bar{H} \bar{W} \bar{H} \bar{L}_{3}+L_{1} M L_{1}+D_{r}^{T} \bar{V} D_{r}\right) \\
& +2 \Gamma \otimes P+\Gamma^{2} \otimes \bar{V}-\Gamma \otimes\left(D_{r}^{T} \bar{V}+\bar{V} D_{r}\right), \\
\Psi_{r 2}= & \Gamma \otimes\left(\bar{V} R_{r 1}\right)+I_{l} \otimes\left(P R_{r 1}-D_{r}^{T} \bar{V} R_{r 1}\right), \\
\Psi_{r 3}= & I_{l} \otimes\left(P R_{r 2}-D_{r}^{T} \bar{V} R_{r 2}\right)+\Gamma \otimes \bar{V} R_{r 2}, \\
\Psi_{r 4}= & \left(\Gamma A_{r}\right) \otimes \bar{V}+A_{r} \otimes\left(P-D_{r}^{T} \bar{V}\right), \\
\Psi_{r 5}= & \left(\Gamma B_{r}\right) \otimes \bar{V}+B_{r} \otimes\left(P-D_{r}^{T} \bar{V}\right), \\
\Psi_{r 6}= & \left(\Gamma C_{r}\right) \otimes \bar{V}+C_{r} \otimes\left(P-D_{r}^{T} \bar{V}\right), \\
\Psi_{r 7}= & I_{l} \otimes\left(R_{r 1}^{T} \bar{V} R_{r 1}-M\right), \\
\Psi_{r 8}= & I_{l} \otimes\left[R_{r 2}^{T} \bar{V} R_{r 2}-(1-\tau) L_{2}^{-1} Q L_{2}^{-1}\right],
\end{aligned}
$$

$$
\begin{aligned}
\Psi_{r 9}= & \left(A_{r}^{T} A_{r}\right) \otimes \bar{V}-\left(1-\tau_{1}\right)\left(I_{m} \otimes U\right), \\
\Psi_{r 10}= & \left(B_{r}^{T} B_{r}\right) \otimes \bar{V}-\left(1-\tau_{2}\right)\left(I_{m} \otimes\left(L_{4}^{-1} V L_{4}^{-1}\right)\right), \\
\Psi_{r 11}= & \left(C_{r}^{T} C_{r}\right) \otimes \bar{V}-I_{m} \otimes \bar{W} \\
\bar{\Psi}_{r 1}= & I_{m} \otimes\left(-\bar{P} \bar{D}_{r}-\bar{D}_{r}^{T} \bar{P}+\bar{Q}+U+L_{3} H W H L_{3}\right. \\
& \left.+\bar{L}_{1} \bar{M} \bar{L}_{1}+\bar{D}_{r}^{T} V \bar{D}_{r}\right) \\
& +2 \bar{\Gamma} \otimes \bar{P}+\bar{\Gamma}^{2} \otimes V-\bar{\Gamma} \otimes\left(\bar{D}_{r}^{T} V+V \bar{D}_{r}\right), \\
\bar{\Psi}_{r 2}= & \bar{\Gamma} \otimes\left(V \bar{R}_{r 1}\right)+I_{m} \otimes\left(\bar{P} \bar{R}_{r 1}-\bar{D}_{r}^{T} V \bar{R}_{r 1}\right), \\
\bar{\Psi}_{3}= & I_{m} \otimes\left(\bar{P}_{R}-\bar{D}_{r}^{T} V \bar{R} r\right)+\bar{\Gamma}_{r 2} \otimes V \bar{R}_{r 2}, \\
\bar{\Psi}_{r 4}= & \left(\bar{\Gamma}_{\bar{A}}\right) \otimes V+\bar{A}_{r} \otimes\left(\bar{P}-\bar{D}_{r}^{T} V\right), \\
\bar{\Psi}_{5}= & \left(\bar{\Gamma}_{B_{r}}\right) \otimes V+\bar{B}_{r} \otimes\left(\bar{P}-\bar{D}_{r}^{T} V\right), \\
\bar{\Psi}_{r 6}= & \left(\bar{\Gamma}_{\bar{C}}\right) \otimes V+\bar{C}_{r} \otimes\left(\bar{P}-\bar{D}_{r}^{T} V\right),
\end{aligned}
$$




$$
\begin{aligned}
& \bar{\Psi}_{r 7}=I_{m} \otimes\left(\bar{R}_{r 1}^{T} V \bar{R}_{r 1}-\bar{M}\right), \\
& \bar{\Psi}_{8}=I_{m} \otimes\left[\bar{R}_{r 2}^{T} V \bar{R}_{r 2}-(1-\sigma) \bar{L}_{2}^{-1} \bar{Q} \bar{L}_{2}^{-1}\right], \\
& \bar{\Psi}_{r 9}=\left(\bar{A}_{r}^{T} \bar{A}_{r}\right) \otimes V-\left(1-\sigma_{1}\right)\left(I_{l} \otimes \bar{U}\right), \\
& \bar{\Psi}_{r 10}=\left(\bar{B}_{r}^{T} \bar{B}_{r}\right) \otimes V-\left(1-\sigma_{2}\right)\left(I_{l} \otimes\left(\bar{L}_{4}^{-1} \bar{V} \bar{L}_{4}\right)\right), \\
& \bar{\Psi}_{r 11}=\left(\bar{C}_{r}^{T} \bar{C}_{r}\right) \otimes V-I_{l} \otimes W .
\end{aligned}
$$

Proof. For the error dynamical system (43), we define the following Lyapunov-Krasovskii function:

$$
\begin{aligned}
& V(t, e(t), \varepsilon(t))=V_{1}(t, e(t), \varepsilon(t))+V_{2}(t, e(t), \varepsilon(t)), \\
& V_{1}=e^{T}(t)\left(I_{l} \otimes P\right) e(t)+\int_{t-\tau(t)}^{t} e^{T}(s)\left(I_{l} \otimes Q\right) e(s) \mathrm{d} s \\
& +\int_{t-\tau_{1}(t)}^{t} \varepsilon^{T}(s)\left(I_{m} \otimes U\right) \varepsilon(s) \mathrm{d} s \\
& +\sum_{j=1}^{m} \sum_{i=1}^{n} w_{i} \int_{0}^{\infty} h_{i}(v) \mathrm{d} v \int_{0}^{\infty} h_{i}(\theta) \\
& \times \int_{t-\theta}^{t} K_{i}^{2}\left(\varepsilon_{j i}(s)\right) \mathrm{d} s \mathrm{~d} \theta \\
& +\int_{t-\tau_{2}(t)}^{t} \dot{\varepsilon}^{T}(s)\left(I_{m} \otimes V\right) \dot{\varepsilon}(s) \mathrm{d} s, \\
& V_{2}=\varepsilon^{T}(t)\left(I_{m} \otimes \bar{P}\right) \varepsilon(t) \\
& +\int_{t-\sigma(t)}^{t} \varepsilon^{T}(s)\left(I_{m} \otimes \bar{Q}\right) \varepsilon(s) \mathrm{d} s \\
& +\int_{t-\sigma_{1}(t)}^{t} e^{T}(s)\left(I_{l} \otimes \bar{U}\right) e(s) \mathrm{d} s \\
& +\sum_{i=1}^{l} \sum_{j=1}^{n} \bar{w}_{j} \int_{0}^{\infty} \bar{h}_{j}(v) \mathrm{d} v \int_{0}^{\infty} \bar{h}_{j}(\theta) \\
& \times \int_{t-\theta}^{t} \bar{K}_{j}\left(e_{i j}(s)\right) \mathrm{d} s \mathrm{~d} \theta \\
& +\int_{t-\sigma_{2}(t)}^{t} \dot{e}^{T}(s)\left(I_{l} \otimes \bar{V}\right) \dot{e}(s) \mathrm{d} s, \\
& \dot{V}_{1}=e^{T}(t)\left(I_{l} \otimes P\right) \dot{e}(t)+\dot{e}^{T}(t)\left(I_{l} \otimes P\right) e(t) \\
& +e^{T}(t)\left(I_{l} \otimes Q\right) e(t)-(1-\dot{\tau}(t)) e^{T}(t-\tau(t)) \\
& \times\left(I_{l} \otimes Q\right) e(t-\tau(t))+\varepsilon^{T}(t)\left(I_{m} \otimes U\right) \varepsilon(t) \\
& -\left(1-\dot{\tau}_{1}(t)\right) \varepsilon^{T}\left(t-\tau_{1}(t)\right)\left(I_{m} \otimes U\right) \varepsilon\left(t-\tau_{1}(t)\right)
\end{aligned}
$$

$$
\begin{aligned}
& +\sum_{j=1}^{m} \sum_{i=1}^{n} w_{i}\left(K_{i}\left(\varepsilon_{j i}(t)\right) \int_{0}^{\infty} h_{i}(v) \mathrm{d} v\right)^{2} \\
& -\sum_{j=1}^{m} \sum_{i=1}^{n} w_{i} \int_{0}^{\infty} h_{i}(v) \mathrm{d} v \int_{0}^{\infty} h_{i}(\theta) K_{i}^{2}\left(\varepsilon_{j i}(t-\theta)\right) \mathrm{d} \theta \\
& +\dot{\varepsilon}^{T}(t)\left(I_{m} \otimes V\right) \dot{\varepsilon}(t) \\
& -\left(1-\dot{\tau}_{2}(t)\right) \dot{\varepsilon}^{T}\left(t-\tau_{2}(t)\right)\left(I_{m} \otimes V\right) \dot{\varepsilon}\left(t-\tau_{2}(t)\right), \\
\dot{V}_{2}= & \varepsilon^{T}(t)\left(I_{m} \otimes \bar{P}\right) \dot{\varepsilon}(t)+\dot{\varepsilon}^{T}(t)\left(I_{m} \otimes \bar{P}\right) \varepsilon(t) \\
& +\varepsilon(t)\left(I_{m} \otimes \bar{Q}\right) \varepsilon(t)-(1-\dot{\sigma}(t)) \varepsilon(t-\sigma(t)) \\
& \times\left(I_{m} \otimes \bar{Q}\right) \varepsilon(t-\sigma(t))+e^{T}(t)\left(I_{l} \otimes \bar{U}\right) e(t) \\
& -\left(1-\dot{\sigma}_{1}(t)\right) e^{T}\left(t-\sigma_{1}(t)\right)\left(I_{l} \otimes \bar{U}\right) e\left(t-\sigma_{1}(t)\right) \\
& \times\left(I_{l} \otimes \bar{V}\right) \dot{e}\left(t-\sigma_{2}(t)\right) . \\
& +\sum_{i=1}^{l} \sum_{j=1}^{n} \bar{w}_{j}\left(\bar{K}_{j}\left(e_{i j}(t)\right) \int_{0}^{\infty} \bar{h}_{j}(v) \mathrm{d} v\right)^{2} \\
& -\sum_{i=1}^{l} \sum_{j=1}^{n} \bar{w}_{j} \int_{0}^{\infty} \bar{h}_{j}(v) \mathrm{d} v \int_{0}^{\infty} \bar{h}_{j}(\theta) \bar{K}_{j}^{2}\left(e_{i j}(t-\theta)\right) \mathrm{d} \theta
\end{aligned}
$$

By (26), we have

$$
\begin{aligned}
& \sum_{j=1}^{m} \sum_{i=1}^{n} w_{i}\left(K_{i}\left(\varepsilon_{j i}(t)\right) \int_{0}^{\infty} h_{i}(v) \mathrm{d} v\right)^{2} \\
& \quad \leq \sum_{j=1}^{m} \varepsilon_{j}^{T}(t) L_{3} H W H L_{3} \varepsilon_{j}(t) \\
& =\varepsilon^{T}(t)\left(I_{m} \otimes\left(L_{3} H W H L_{3}\right)\right) \varepsilon(t) .
\end{aligned}
$$

Using (30), we get

$$
\begin{gathered}
\sum_{j=1}^{m} \sum_{i=1}^{n} w_{i} \int_{0}^{\infty} h_{i}(v) \mathrm{d} v \int_{0}^{\infty} h_{i}(\theta) K_{i}^{2}\left(\varepsilon_{j i}(s)(t-\theta)\right) \mathrm{d} \theta \\
\geq \sum_{j=1}^{m}\left(\int_{-\infty}^{t} h(t-s) K\left(\varepsilon_{j}(s)\right) \mathrm{d} s\right)^{T} \\
\quad \times W \int_{-\infty}^{t} h(t-s) K\left(\varepsilon_{j}(s)\right) \mathrm{d} s \\
=\left(\int_{-\infty}^{t}\left(I_{m} \otimes h(t-s)\right) \widetilde{K}(\varepsilon(s)) \mathrm{d} s\right)^{T}\left(I_{m} \otimes W\right) \\
\quad \times \int_{-\infty}^{t}\left(I_{m} \otimes h(t-s)\right) \widetilde{K}(\varepsilon(s)) \mathrm{d} s .
\end{gathered}
$$


From $\left(S_{3}\right)$ and (46)-(48), we have

$$
\begin{aligned}
\dot{V}_{1} \leq & e^{T}(t)\left(I_{l} \otimes P\right) \dot{e}(t)+\dot{e}^{T}(t)\left(I_{l} \otimes P\right) e(t) \\
& +e^{T}(t)\left(I_{l} \otimes Q\right) e(t)-(1-\tau) e^{T}(t-\tau(t)) \\
& \times\left(I_{l} \otimes Q\right) e(t-\tau(t)) \\
& +\varepsilon^{T}(t)\left[I_{m} \otimes\left(U+L_{3} H W H L_{3}\right)\right] \varepsilon(t) \\
& -\left(1-\tau_{1}\right) \varepsilon^{T}\left(t-\tau_{1}(t)\right)\left(I_{m} \otimes U\right) \varepsilon\left(t-\tau_{1}(t)\right) \\
& -\left(\int_{-\infty}^{t}\left(I_{m} \otimes h(t-s)\right) \widetilde{K}(\varepsilon(s)) \mathrm{d} s\right)^{T}\left(I_{m} \otimes W\right) \\
& \times \int_{-\infty}^{t}\left(I_{m} \otimes h(t-s)\right) \widetilde{K}(\varepsilon(s)) \mathrm{d} s \\
& +\dot{\varepsilon}^{T}(t)\left(I_{m} \otimes V\right) \dot{\varepsilon}(t)-\left(1-\tau_{2}\right) \dot{\varepsilon}^{T}\left(t-\tau_{2}(t)\right) \\
& \times\left(I_{m} \otimes V\right) \dot{\varepsilon}\left(t-\tau_{2}(t)\right) .
\end{aligned}
$$

In the same way, we have

$$
\begin{aligned}
\dot{V}_{2} \leq & \varepsilon^{T}(t)\left(I_{m} \otimes \bar{P}\right) \dot{\varepsilon}(t)+\dot{\varepsilon}^{T}(t)\left(I_{l} \otimes \bar{P}\right) \varepsilon(t) \\
& +\varepsilon^{T}(t)\left(I_{m} \otimes \bar{Q}\right) \varepsilon(t)-(1-\sigma) \varepsilon^{T}(t-\sigma(t)) \\
& \times\left(I_{m} \otimes Q\right) \varepsilon(t-\sigma(t)) \\
& +e^{T}(t)\left[I_{l} \otimes\left(\bar{U}+\bar{L}_{3} \bar{H} \bar{W} \bar{H} \bar{L}_{3}\right)\right] e(t) \\
& -\left(1-\sigma_{1}\right) e^{T}\left(t-\sigma_{1}(t)\right)\left(I_{l} \otimes \bar{U}\right) e\left(t-\sigma_{1}(t)\right) \\
& -\left(\int_{-\infty}^{t}\left(I_{l} \otimes \bar{h}(t-s)\right) \widehat{K}(e(s)) \mathrm{d} s\right)^{T}\left(I_{l} \otimes \bar{W}\right) \\
& \times \int_{-\infty}^{t}\left(I_{l} \otimes \bar{h}(t-s)\right) \widehat{K}(e(s)) \mathrm{d} s \\
& +\dot{e}^{T}(t)\left(I_{l} \otimes \bar{V}\right) \dot{e}(t)-\left(1-\sigma_{2}\right) \dot{e}^{T}\left(t-\sigma_{2}(t)\right) \\
& \times\left(I_{l} \otimes \bar{V}\right) \dot{e}\left(t-\sigma_{2}(t)\right) .
\end{aligned}
$$

From $\left(S_{1}\right)$ and $\left(S_{2}\right)$,

$$
\begin{aligned}
& e^{T}(t) {\left[I_{l} \otimes\left(L_{1} M L_{1}\right)\right] e(t) } \\
&-\widetilde{F}_{1}^{T}(e(t))\left(I_{l} \otimes M\right) \widetilde{F}_{1}(e(t)) \geq 0, \\
& \varepsilon^{T}(t) {\left[I_{m} \otimes\left(\bar{L}_{1} \bar{M} \bar{L}_{1}\right)\right] \varepsilon(t) } \\
&-\widehat{F}_{1}^{T}(\varepsilon(t))\left(I_{m} \otimes \bar{M}\right) \widehat{F}_{1}(\varepsilon(t)) \geq 0 \\
& e^{T}(t-\tau(t))\left(I_{l} \otimes Q\right) e(t-\tau(t)) \\
& \geq \widetilde{F}_{2}^{T}(e(t-\tau(t)))\left[I_{l} \otimes\left(L_{2}^{-1} Q L_{2}^{-1}\right)\right] \widetilde{F}_{2}(e(t-\tau(t))),
\end{aligned}
$$

$$
\begin{aligned}
& \varepsilon^{T}(t-\sigma(t))\left(I_{m} \otimes \bar{Q}\right) \varepsilon(t-\sigma(t)) \\
& \quad \geq \widehat{F}_{2}^{T}(\varepsilon(t-\sigma(t)))\left[I_{m} \otimes\left(\bar{L}_{2}^{-1} \bar{Q} \bar{L}_{2}^{-1}\right)\right] \widehat{F}_{2}(\varepsilon(t-\sigma(t))), \\
& \dot{\varepsilon}^{T}\left(t-\tau_{2}(t)\right)\left(I_{m} \otimes V\right) \dot{\varepsilon}\left(t-\tau_{2}(t)\right) \\
& \quad \geq \widetilde{G}^{T}\left(\dot{\varepsilon}\left(t-\tau_{2}(t)\right)\right)\left(I_{m} \otimes\left(L_{4}^{-1} V L_{4}^{-1}\right)\right) \widetilde{G}\left(\dot{\varepsilon}\left(t-\tau_{2}(t)\right)\right), \\
& \dot{e}^{T}\left(t-\sigma_{2}(t)\right)\left(I_{l} \otimes \bar{V}\right) \dot{e}\left(t-\sigma_{2}(t)\right) \\
& \quad \geq \widehat{G}^{T}\left(\dot{e}\left(t-\sigma_{2}(t)\right)\right)\left(I_{l} \otimes\left(\bar{L}_{4}^{-1} \bar{V} \bar{L}_{4}^{-1}\right)\right) \widehat{G}\left(\dot{e}\left(t-\sigma_{2}(t)\right)\right) .
\end{aligned}
$$

With the aid of (43) and (51), we have

$$
\dot{V} \leq \sum_{r=1}^{N} \xi_{r}(t, \lambda)\left[\eta^{T}(t) \Omega_{r} \eta(t)+\bar{\eta}^{T}(t) \bar{\Omega}_{r} \bar{\eta}(t)\right]
$$

where

$$
\begin{gathered}
\eta(t)=\left(e^{T}(t), \widetilde{F}_{1}^{T}(e(t)), \widetilde{F}_{2}^{T}(e(t-\tau(t))), \varepsilon^{T}\left(t-\tau_{1}(t)\right),\right. \\
\widetilde{G}^{T}\left(\dot{\varepsilon}\left(t-\tau_{2}(t)\right)\right), \\
\left.\left(\int_{-\infty}^{t}\left(I_{m} \otimes h(t-s)\right) \widetilde{\mathrm{K}}(\varepsilon(s)) \mathrm{d} s\right)^{T}\right)^{T}, \\
\bar{\eta}(t)=\left(\varepsilon^{T}(t), \widehat{F}_{1}^{T}(\varepsilon(t)), \widehat{F}_{2}^{T}(\varepsilon(t-\sigma(t))), e^{T}\left(t-\sigma_{1}(t)\right),\right. \\
\widehat{G}^{T}\left(\dot{e}\left(t-\sigma_{2}(t)\right)\right), \\
\left.\left(\int_{-\infty}^{t}\left(I_{l} \otimes \bar{h}(t-s)\right) \widehat{\mathrm{K}}(e(s)) \mathrm{d} s\right)^{T}\right)^{T} .
\end{gathered}
$$

Let $\rho=\min \left\{\rho_{1}, \rho_{2}\right\}$, where $\rho_{1}=-\min \left\{\rho_{\min }\left(\Omega_{r}\right), r \in\right.$ $\aleph\}, \rho_{2}=-\min \left\{\rho_{\min }\left(\bar{\Omega}_{r}\right), r \in \aleph\right\}$, then $\rho>0$ and

$$
\begin{aligned}
\dot{V} & \leq-\rho_{1} \sum_{i=1}^{l} e_{i}^{T}(t) e_{i}(t)-\rho_{2} \sum_{j=1}^{m} \varepsilon_{j}^{T}(t) \varepsilon_{j}(t) \\
& \leq-\rho\left[\sum_{i=1}^{l} e_{i}^{T}(t) e_{i}(t)+\sum_{j=1}^{m} \varepsilon_{j}^{T}(t) \varepsilon_{j}(t)\right] .
\end{aligned}
$$

The following proof is similar to that of Theorem 4 and is omitted here.

\section{Simulations}

In this section, numerical examples are provided to demonstrate the validity of the synchronization criteria obtained 
in the previous sections. Consider the following network as drive network:

$$
\begin{aligned}
& \dot{x}_{i}(t)=\sum_{r=1}^{N} \xi_{r}(t, \lambda) \\
& \times\left[-D_{r} x_{i}+R_{r 1} f_{1}\left(x_{i}(t)\right)\right. \\
& +R_{r 2} f_{2}\left(x_{i}(t-\tau(t))\right) \\
& +I_{r}+\sum_{j=1}^{m} a_{r i j} y_{j}\left(t-\tau_{1}(t)\right) \\
& +\sum_{j=1}^{m} b_{r i j} g\left(\dot{y}_{j}\left(t-\tau_{2}(t)\right)\right) \\
& \left.+\sum_{j=1}^{m} c_{r i j} \int_{-\infty}^{t} h(t-s) k\left(y_{j}(s)\right) \mathrm{d} s\right], \\
& i=12, \ldots, l \text {, } \\
& \dot{y}_{j}(t)=\sum_{r=1}^{N} \xi_{r}(t, \lambda) \\
& \times\left[-\bar{D}_{r} y_{j}+\bar{R}_{r 1} \bar{f}_{1}\left(y_{j}(t)\right)\right. \\
& +\bar{R}_{r 2} \bar{f}_{2}\left(y_{j}(t-\sigma(t))\right) \\
& +J_{r}+\sum_{i=1}^{l} \bar{a}_{r j i} x_{i}\left(t-\sigma_{1}(t)\right) \\
& +\sum_{i=1}^{l} \bar{b}_{r j i} \bar{g}\left(\dot{x}_{i}\left(t-\sigma_{2}(t)\right)\right) \\
& \left.+\sum_{i=1}^{l} \bar{c}_{r j i} \int_{-\infty}^{t} \bar{h}(t-s) \bar{k}\left(x_{i}(s)\right) \mathrm{d} s\right], \\
& j=1,2, \ldots, m \text {, }
\end{aligned}
$$

where $x_{i}(t), y_{j}(t) \in \mathrm{R}^{2}, l=3$, and $m=3 . f_{1}(z(t))=$ $0.1\left(\tanh \left(z_{1}(t)\right), \tanh \left(z_{2}(t)\right)\right)^{T}, z(t)=\left(z_{1}(t), z_{2}(t)\right)^{T}, \bar{f}_{1}=$ $g=\bar{g}=k=\bar{k}=f_{2}=\bar{f}_{2}=f_{1}$, and $h(t)=\bar{h}(t)=\operatorname{diag}\left(e^{-t}\right.$, $\left.e^{-t}\right)$. Choose time delays $\tau(t)=1+0.4 \sin t, \tau_{1}(t)=2+$ $0.2 \arctan (t), \tau_{2}(t)=0.6+0.5 \cos t$, and $\sigma(t)=1+0.8 \sin t$, $\sigma_{1}(t)=0.7+0.1 \cos t, \sigma_{2}(t)=0.5+\left(0.3 e^{t} /\left(1+e^{t}\right)\right)$. We define a switching rule $\lambda: t \in[0,+\infty) \rightarrow\{1,2\}, \lambda(t)=$ $\operatorname{int}(t) \bmod 2+1$. The other parameters are as follows:

$$
\begin{array}{rlr}
D_{1} & =\left(\begin{array}{cc}
1.8 & 0 \\
0 & 4
\end{array}\right), & R_{11}=\left(\begin{array}{cc}
-1 & 1 \\
0 & 0.2
\end{array}\right), \\
R_{12}=\left(\begin{array}{cc}
1 & 0.5 \\
0.6 & -1
\end{array}\right), & I_{1}=J_{1}=(1,2)^{T},
\end{array}
$$

$$
\begin{aligned}
& A_{1}=\left(a_{1 i j}\right)=\left(\begin{array}{ccc}
-2 & -2 & 0 \\
0 & 2 & -2 \\
1 & 1 & -2
\end{array}\right) \\
& B_{1}=\left(b_{1 i j}\right)=\left(\begin{array}{ccc}
-0.2 & 0 & -0.2 \\
0.1 & -0.4 & 0.3 \\
0.2 & 0.1 & -0.3
\end{array}\right) \text {, } \\
& C_{1}=\left(c_{1 i j}\right)=\left(\begin{array}{ccc}
1 & -1 & 0 \\
1 & 1 & -2 \\
-1 & 0 & 1
\end{array}\right) \text {, } \\
& \bar{D}_{1}=\left(\begin{array}{ll}
2 & 0 \\
0 & 1
\end{array}\right), \quad \bar{R}_{11}=\left(\begin{array}{cc}
-0.3 & 1 \\
0.2 & 0.3
\end{array}\right) \text {, } \\
& \bar{R}_{12}=\left(\begin{array}{cc}
0.3 & 0.4 \\
0.6 & -0.5
\end{array}\right), \quad \bar{A}_{1}=\left(\bar{a}_{1 j i}\right)=\left(\begin{array}{ccc}
4 & 0 & -4 \\
1 & 1 & -2 \\
1 & 0 & -1
\end{array}\right) \text {, } \\
& \bar{B}_{1}=\left(\bar{b}_{1 j i}\right)=\left(\begin{array}{ccc}
0.1 & 0 & -0.1 \\
0.2 & -0.3 & 0.1 \\
0.1 & 0.2 & -0.2
\end{array}\right) \text {, } \\
& \bar{C}_{1}=\left(\bar{c}_{1 j i}\right)=\left(\begin{array}{ccc}
-3 & 1 & 2 \\
2 & 0 & -2 \\
-4 & 0 & 4
\end{array}\right), \\
& D_{2}=\left(\begin{array}{cc}
1.4 & 0 \\
0 & 1.4
\end{array}\right), \quad R_{21}=\left(\begin{array}{cc}
1 & -1 \\
-5 & 3
\end{array}\right) \text {, } \\
& R_{22}=\left(\begin{array}{cc}
-1.5 & -0.1 \\
-3 & -1
\end{array}\right), \quad A_{2}=\left(a_{2 i j}\right)=\left(\begin{array}{ccc}
-1 & 1 & 0 \\
0 & 2 & -2 \\
1.2 & 1 & -2.2
\end{array}\right) \text {, } \\
& B_{2}=\left(b_{2 i j}\right)=\left(\begin{array}{ccc}
0.1 & -0.1 & 0 \\
0.1 & -0.5 & 0.4 \\
0.2 & 0 & -0.2
\end{array}\right) \text {, } \\
& C_{2}=\left(c_{2 i j}\right)=\left(\begin{array}{ccc}
2 & -2 & 0 \\
3 & -1 & -2 \\
0 & -1 & 1
\end{array}\right), \\
& \bar{D}_{2}=\left(\begin{array}{cc}
1.2 & 0 \\
0 & 1.2
\end{array}\right), \quad \bar{R}_{21}=\left(\begin{array}{cc}
-0.3 & 1 \\
-4 & 1
\end{array}\right), \\
& \bar{R}_{22}=\left(\begin{array}{cc}
0.3 & 0.4 \\
-2 & -1
\end{array}\right), \quad I_{2}=J_{2}=(3,4)^{T}, \\
& \bar{A}_{2}=\left(\bar{a}_{2 j i}\right)=\left(\begin{array}{ccc}
1 & -1 & 0 \\
1 & 2 & -3 \\
1 & 1 & -2
\end{array}\right), \\
& \bar{B}_{2}=\left(\bar{b}_{2 j i}\right)=\left(\begin{array}{ccc}
0.2 & -0.2 & 0 \\
0.1 & -0.2 & 0.1 \\
0.3 & 0.1 & -0.4
\end{array}\right) \text {, } \\
& \bar{C}_{2}=\left(\bar{c}_{2 j i}\right)=\left(\begin{array}{ccc}
-5 & 1 & 4 \\
1 & 1 & -2 \\
-1 & 0 & 1
\end{array}\right) .
\end{aligned}
$$


The response network of drive network (55) is

$$
\begin{aligned}
& \dot{\hat{x}}_{i}(t)=\sum_{r=1}^{N} \xi_{r}(t, \lambda) \\
& \times\left[-D_{r} \widehat{x}_{i}(t)+R_{r 1} f_{1}\left(\widehat{x}_{i}(t)\right)\right. \\
& +R_{r 2} f_{2}\left(\widehat{x}_{i}(t-\tau(t))\right) \\
& +I_{r}+\sum_{j=1}^{m} a_{r i j} \widehat{y}_{j}\left(t-\tau_{1}(t)\right) \\
& +\sum_{j=1}^{m} b_{r i j} g\left(\dot{\hat{y}}_{j}\left(t-\tau_{2}(t)\right)\right) \\
& \left.+\sum_{j=1}^{m} c_{r i j} \int_{-\infty}^{t} h(t-s) k\left(\hat{y}_{j}(s)\right) \mathrm{d} s+u_{i}(t)\right], \\
& \dot{\hat{y}}_{j}(t)=\sum_{r=1}^{N} \xi_{r}(t, \lambda) \\
& \times\left[-\bar{D}_{r} \widehat{y}_{j}(t)+\bar{R}_{r 1} \bar{f}_{1}\left(\widehat{y}_{j}(t)\right)\right. \\
& +\bar{R}_{r 2} \bar{f}_{2}\left(\widehat{y}_{j}(t-\sigma(t))\right) \\
& +J_{r}+\sum_{i=1}^{l} \bar{a}_{r j i} \widehat{x}_{i}\left(t-\sigma_{1}(t)\right) \\
& +\sum_{i=1}^{l} \bar{b}_{r j i} \bar{g}\left(\dot{\hat{x}}_{i}\left(t-\sigma_{2}(t)\right)\right) \\
& \left.+\sum_{i=1}^{l} \bar{c}_{r j i} \int_{-\infty}^{t} \bar{h}(t-s) \bar{k}\left(\widehat{x}_{i}(s)\right) \mathrm{d} s+v_{j}(t)\right],
\end{aligned}
$$

where $u_{i}(t), v_{j}(t) \in \mathrm{R}^{2}$.

Let $\gamma_{1}=\gamma_{2}=\gamma_{3}=15, \eta_{1}=\eta_{2}=\eta_{3}=16, \alpha=0.5, \beta=0.5$, and the feasible solution of the matrix inequalities (15)-(19) by employing MATLAB LMI Toolbox be as follows:

$$
\begin{gathered}
p=7.5267, \quad \bar{p}=7.8951 \\
P=\left(\begin{array}{cc}
12.9508 & 0.2817 \\
0.2817 & 10.7871
\end{array}\right), \quad Q=\left(\begin{array}{cc}
83.6405 & 3.6674 \\
3.6674 & 68.8593
\end{array}\right) \\
U=\left(\begin{array}{cc}
119.1080 & 0.2216 \\
0.2216 & 55.7869
\end{array}\right) \\
W=\left(\begin{array}{cc}
233.7870 & 0 \\
0 & 223.7801
\end{array}\right)
\end{gathered}
$$

$$
\begin{aligned}
& M_{1}=\left(\begin{array}{cc}
227.1153 & 0 \\
0 & 202.1669
\end{array}\right), \\
& M_{2}=\left(\begin{array}{cc}
206.3034 & 0 \\
0 & 217.8341
\end{array}\right), \\
& M_{3}=\left(\begin{array}{cc}
86.9723 & 0 \\
0 & 41.4980
\end{array}\right), \quad \bar{P}=\left(\begin{array}{cc}
19.4548 & 0.0336 \\
0.0336 & 11.6060
\end{array}\right), \\
& \bar{Q}=\left(\begin{array}{ccc}
26.1973 & 0.5411 \\
0.5411 & 9.6902
\end{array}\right), \quad \bar{U}=\left(\begin{array}{cc}
30.9126 & 0.9850 \\
0.9850 & 22.6634
\end{array}\right), \\
& \bar{W}=\left(\begin{array}{cc}
316.6943 & 0 \\
0 & 287.9793
\end{array}\right), \\
& \bar{M}_{1}=\left(\begin{array}{cc}
183.0419 & 0 \\
0 & 162.4080
\end{array}\right), \\
& \bar{M}_{2}=\left(\begin{array}{cc}
250.2787 & 0 \\
0 & 409.0398
\end{array}\right), \\
& \bar{M}_{3}=\left(\begin{array}{cc}
13.8704 & 0 \\
0 & 10.1694
\end{array}\right) .
\end{aligned}
$$

The initial values are chosen as $x_{i}(s)=(-5,9), y_{j}(s)=(-6$, $7)^{T}, \widehat{x}_{i}(s)=2 i(2,5)^{T}, \hat{y}_{j}(s)=3 j(2,-1)^{T}$, and $s \in[-2,0]$. Clearly, the two coupled networks (55) and (57) satisfy the conditions of Theorem 4 . Figure 1 presents the synchronization errors of the state variables between the two networks. The simulation result shows that the synchronization is achieved under the proposed controllers (20). Thus, the proposed synchronization control scheme in Theorem 4 is valid.

Let $\gamma_{1}=\gamma_{2}=\gamma_{3}=12, \bar{\gamma}_{1}=\bar{\gamma}_{2}=\bar{\gamma}_{3}=17$, then the feasible solution of the matrix inequalities (44) in Theorem 8 by employing MATLAB LMI Toolbox is as follows:

$$
\begin{gathered}
P=\left(\begin{array}{ll}
0.0251 & 0.0005 \\
0.0005 & 0.0214
\end{array}\right), \quad U=\left(\begin{array}{cc}
3.7826 & -0.0043 \\
-0.0043 & 3.5381
\end{array}\right), \\
Q=\left(\begin{array}{cc}
4.2792 & 0 \\
0 & 3.7789
\end{array}\right), \\
V=\left(\begin{array}{cc}
0.0116 & 0 \\
0 & 0.0079
\end{array}\right), \quad M=\left(\begin{array}{cc}
3.4521 & 0 \\
0 & 3.1482
\end{array}\right), \\
W=\left(\begin{array}{cc}
3.7071 & 0 \\
0 & 3.3433
\end{array}\right), \quad \bar{P}=\left(\begin{array}{cc}
0.0298 & 0 \\
0 & 0.0268
\end{array}\right), \\
\bar{U}=\left(\begin{array}{cc}
3.3712 & -0.0132 \\
-0.0132 & 3.1782
\end{array}\right), \quad \bar{Q}=\left(\begin{array}{cc}
7.2228 & 0 \\
0 & 6.3479
\end{array}\right), \\
\bar{V}=\left(\begin{array}{cc}
0.0077 & 0 \\
0 & 0.0052
\end{array}\right), \quad \bar{M}=\left(\begin{array}{cc}
3.3924 & 0 \\
0 & 3.0142
\end{array}\right), \\
\bar{W}=\left(\begin{array}{cc}
3.4120 & 0 \\
0 & 3.2474
\end{array}\right) .
\end{gathered}
$$




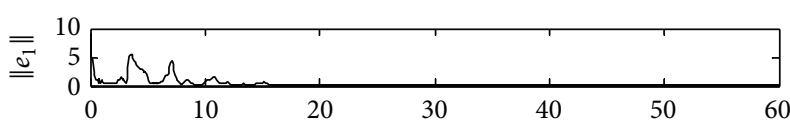

(a)

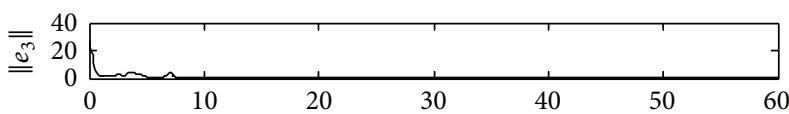

(c)

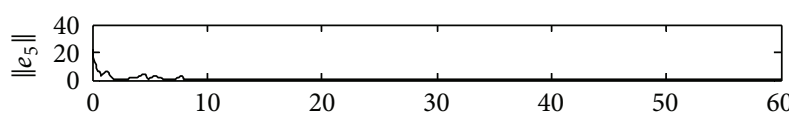

(e)

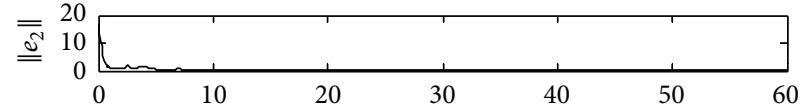

(b)

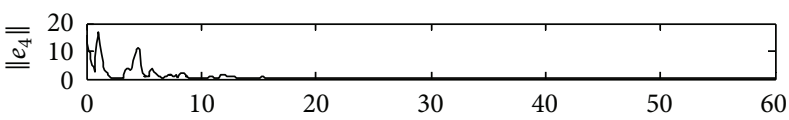

(d)

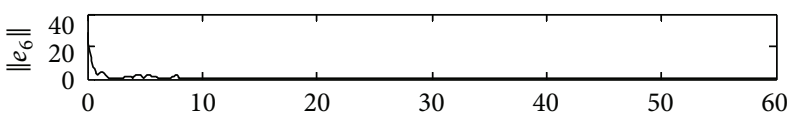

(f)

FIGURE 1: Synchronization errors of BDN (55) and (57) with adaptive feedback controllers (20).

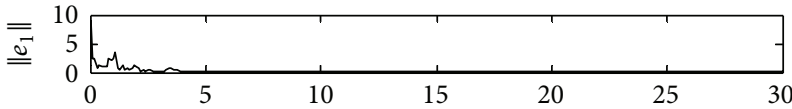

(a)

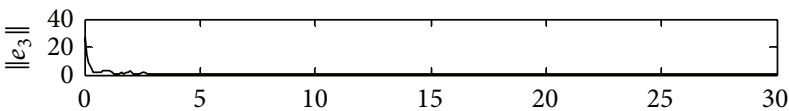

(c)

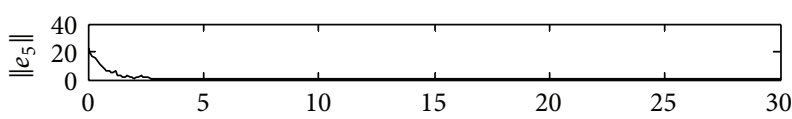

(e)

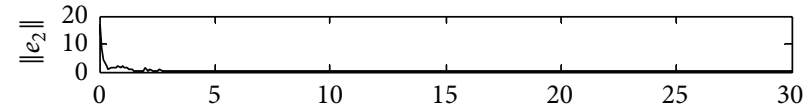

(b)

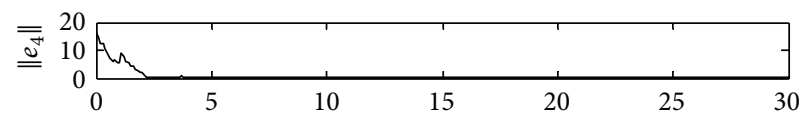

(d)

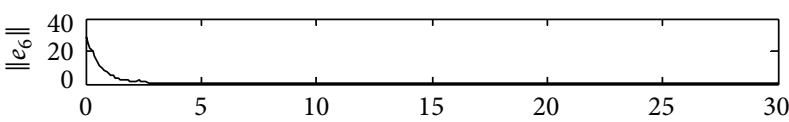

(f)

FIGURE 2: Synchronization errors with adaptive feedback controllers (41).

Using the controllers (41), the simulation result is given in Figure 2, which shows that the proposed synchronization control scheme in Theorem 8 is effective.

\section{Conclusions}

In this paper, we have proposed a general SCBNN with distributed delays and derivative coupling and investigated the synchronization problem in the two coupled SCBNNs. Using linear matrix inequality (LMI) approach and Barbǎlat lemma, we have deviated some useful synchronization criteria to ensure the synchronization of these two SCBNNs by constructing effective controllers. Compared with relative previous jobs, the controllers proposed by us are more simple and feasible. Some simulation results have been presented to demonstrate our theoretical results. In our future work, we will consider using pinning control to realize the synchronization of SCBNNs and identify the network topology of the unknown SCBNNs.

\section{Acknowledgments}

This research is supported by the National Natural Science Foundation of China under Grants nos. 61272530 and 11072059, the Natural Science Foundation of Jiangsu Province of China under Grants no. BK2012741, Specialized Research Fund for the Doctoral Program of Higher Education under Grants no. 20110092110017, and the National Social Science Fund of China under Grant no. 11BGL039.

\section{References}

[1] I. Tokuda, T. Nagashima, and K. Aihara, "Global bifurcation structure of chaotic neural networks and its application to traveling salesman problems," Neural Networks, vol. 10, no. 9, pp. 1673-1690, 1997.

[2] Y. F. Zhang and Z. Y. He, "A secure communication scheme based on cellular neural networks," Proceedings of the IEEE International Conference on Intelligent Processing Systems, vol. 1, pp. 521-524, 1997. 
[3] T. Kwok and K. A. Smith, "Experimental analysis of chaotic neural network models for combinatorial optimization under a unifying framework," Neural Networks, vol. 13, no. 7, pp. 731$744,2000$.

[4] B. Kosko, "Adaptive bidirectional associative memories," Applied Optics, vol. 26, no. 23, pp. 4947-4960, 1987.

[5] B. Kosko, "Bi-directional associative memories," IEEE Transactions on Systems, Man and Cybernetics, vol. 18, no. 1, pp. 49-60, 1988.

[6] J. Liang and J. Cao, "Exponential stability of continuous-time and discrete-time bidirectional associative memory networks with delays," Chaos, Solitons and Fractals, vol. 22, no. 4, pp. 773785, 2004.

[7] S. Arik, "Global asymptotic stability analysis of bidirectional associative memory neural networks with time delays," IEEE Transactions on Neural Networks, vol. 16, no. 3, pp. 580-586, 2005.

[8] J. H. Ge and J. Xu, "Synchronization and synchronized periodicsolution in a simplified five-neuron BAM neural network with delays," Neurocomputing, vol. 74, no. 6, pp. 993-999, 2011.

[9] B. Zhou and Q. K. Song, "Stability and Hopf bifurcation analysis of a tri-neuron BAM neural network with distributed delay," Neurocomputing, vol. 82, no. 1, pp. 69-83, 2012.

[10] D. Liberzon and A. S. Morse, "Basic problems in stability and design of switched systems," IEEE Control Systems Magazine, vol. 19, no. 5, pp. 59-70, 1999.

[11] J. Zhao and M. W. Spong, "Hybrid control for global stabilization of the cart-pendulum system," Automatica, vol. 37, no. 12, pp. 1941-1951, 2001.

[12] K. S. Narendra, O. A. Driollet, M. Feiler, and K. George, "Adaptive control using multiple models, switching and tuning," International Journal of Adaptive Control and Signal Processing, vol. 17, no. 2, pp. 87-102, 2003.

[13] D. Liberzon, Switching in Systems and Control, Birkhauser, Boston, Mass, USA, 2003.

[14] Z. D. Sun and S. S. Ge, "Analysis and synthesis of switched linear control systems," Automatica, vol. 41, no. 2, pp. 181-195, 2005.

[15] Y. Tsividis, "Switched neural networks," United States Patent, Patent number 4873661, 1989.

[16] T. X. Brown, "Neural networks for switching," IEEE Communications Magazine, vol. 27, no. 11, pp. 72-81, 1989.

[17] M. Muselli, Gene Selection Through Switched Neural Networks, Contribution to NETTAB 03, Bologna, Italy, 2003.

[18] D. Ding, Z. Wang, B. Shen, and H. Shu, "H-infinity state estimation for discrete-time complex networks with randomly occurring sensor saturations and randomly varying sensor delays," IEEE Transactions on Neural Networks and Learning Systems, vol. 23, no. 5, pp. 725-736, 2012.

[19] V. Perez-Munuzuri, V. Perez-Villar, and L. O. Chua, "Autowaves for image processing on a two-dimensional CNN array of excitable nonlinear circuits: flat and wrinkled labyrinths," IEEE Transactions on Circuits and Systems I, vol. 40, no. 3, pp. 174-181, 1993.

[20] B. Hen, Z. Wang, Y. Hung, and G. YChesi, "Distributed Hinfinity state estimation with stochastic parameters and nonlinearities through sensor networks: the finite-horizon case," Automatica, vol. 48, no. 8, pp. 1575-1585, 2012.

[21] M. E. J. Newman, "Scientific collaboration networks. II. Shortest paths, weighted networks, and centrality," Physical Review E, vol. 64, no. 1, Article ID 016132, 2001.
[22] D. H. Wang, L. Zhou, and Z. R. Di, "Bipartite producer-consumer networks and the size distribution of firms," Physica A, vol. 363, no. 2, pp. 359-366, 2006.

[23] Z. Jia, H. Wang, J. Wang, and Y. Li, “The weighted identification of a bipartite-graph complex dynamicalnetwork based on adaptive synchronization," Control Theory \& Applications, vol. 27, no. 1, pp. 107-110, 2010.

[24] W. L. Lu and T. P. Chen, "Synchronization of coupled connected neural networks with delays," IEEE Transactions on Circuits and Systems I, vol. 51, no. 12, pp. 2491-2503, 2004.

[25] L. Wang, H. P. Dai, H. Dong, Y. H. Shen, and Y. X. Sun, "Adaptive synchronization of weighted complex dynamical networks with coupling time-varying delays," Physics Letters A, vol. 372, no. 20, pp. 3632-3639, 2008.

[26] H. Dong, Z. Wang, J. Lam, and H. Gao, "Fuzzy-model-based robust fault detection with stochastic mixed time-delays and successive packet dropouts," IEEE Transactions on Systems, Man, and Cybernetics B, vol. 42, no. 2, pp. 365-376, 2012.

[27] T. Liu, J. Zhao, and D. J. Hill, "Synchronization of complex delayed dynamical networks with nonlinearly coupled nodes," Chaos, Solitons and Fractals, vol. 40, no. 3, pp. 1506-1519, 2009.

[28] Y. H. Xu, W. N. Zhou, J. A. Fang, and W. Sun, "Adaptive synchronization of the complex dynamical network with non-derivative and derivative coupling," Physics Letters A, vol. 374, no. 15-16, pp. 1673-1677, 2010.

[29] Q. X. Bian and H. X. Yao, "Adaptive synchronization of bipartite dynamical networks with distributed delays and nonlinear derivative coupling," Communications in Nonlinear Science and Numerical Simulation, vol. 16, no. 10, pp. 4089-4098, 2011.

[30] B. Shen, Z. D. Wang, and X. H. Liu, "Sampled-data synchronization control of complex dynamical networks with stochastic sampling," IEEE Transactions on Automatic Control, vol. 57, no. 10, pp. 2644-2650, 2012.

[31] A. Arenas, A. Díaz-Guilera, J. Kurths, Y. Moreno, and C. S. Zhou, "Synchronization in complex networks," Physics Reports, vol. 469, no. 3, pp. 93-153, 2008.

[32] G. Solís-Perales, E. Ruiz-Velázquez, and D. Valle-Rodríguez, "Synchronization in complex networks with distinct chaotic nodes," Communications in Nonlinear Science and Numerical Simulation, vol. 14, no. 6, pp. 2528-2535, 2009.

[33] W. W. Yu, J. D. Cao, and W. L. Lu, "Synchronization control of switched linearly coupled neural networks with delay," Neurocomputing, vol. 73, no. 4-6, pp. 858-866, 2010.

[34] L. Huang, Linear Algebra in Systems and Control Theory, Science Press, Beijing, China, 1984.

[35] E. N. Sanchez and J. P. Perez, "Input-to-state stability analysis for dynamic neural networks," IEEE Transactions on Circuits and Systems I, vol. 46, no. 11, pp. 1395-1398, 1999.

[36] V. M. Popov, Hyperstability of Control Systems, Springer, NewYork, NY, USA, 1973. 


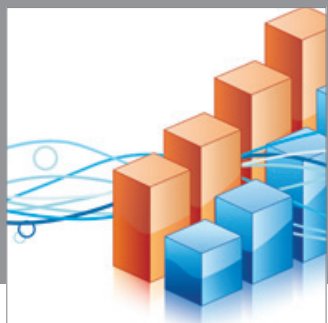

Advances in

Operations Research

mansans

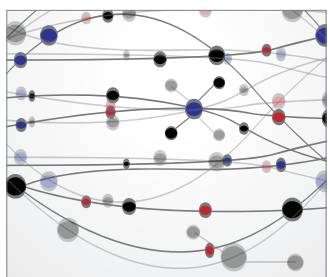

The Scientific World Journal
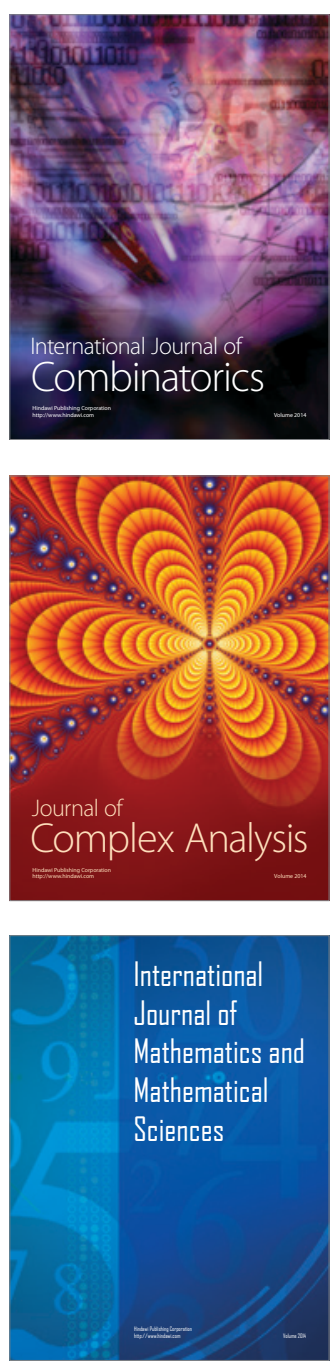
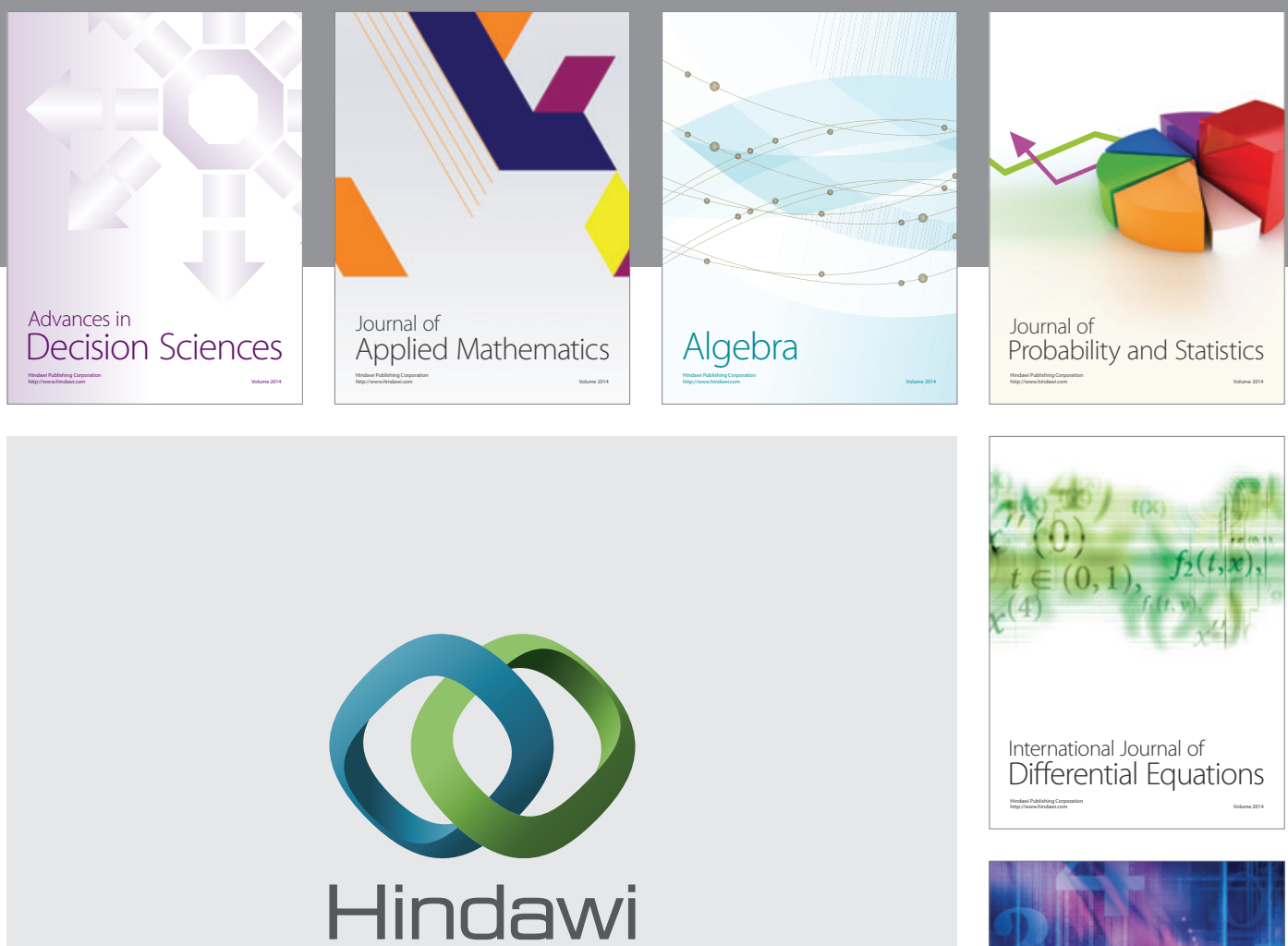

Submit your manuscripts at http://www.hindawi.com
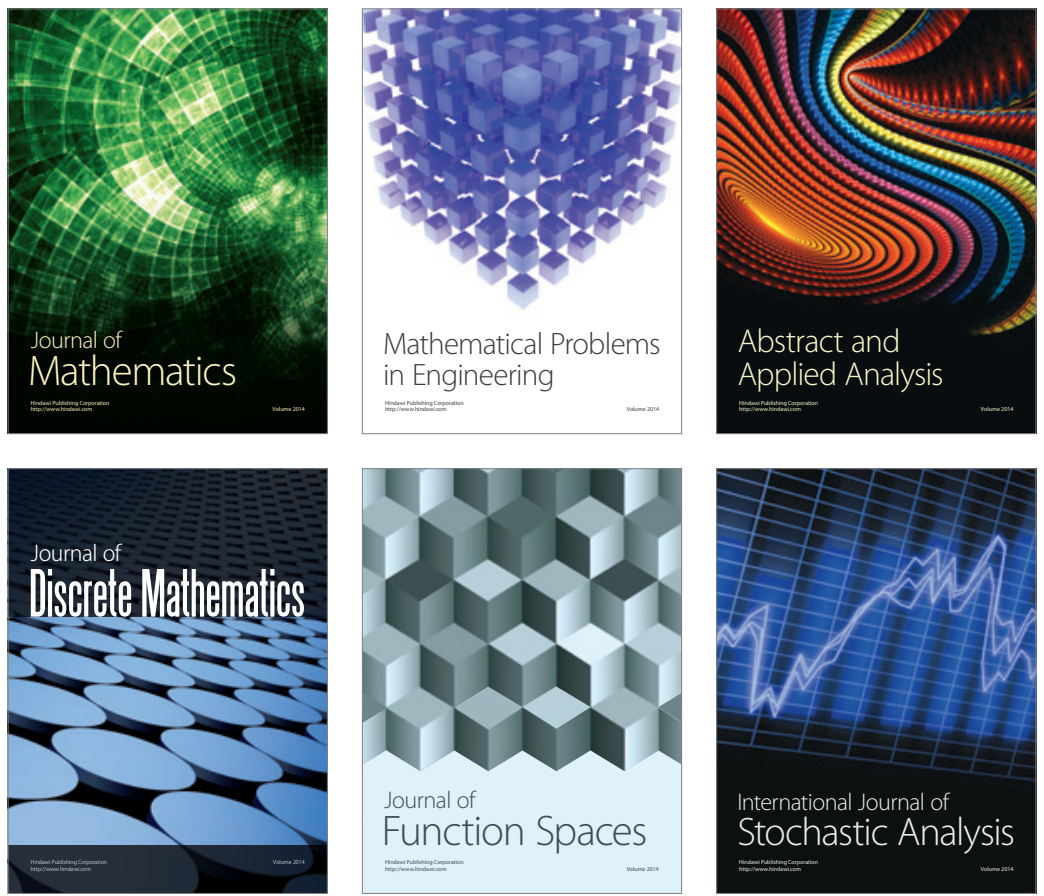

Journal of

Function Spaces

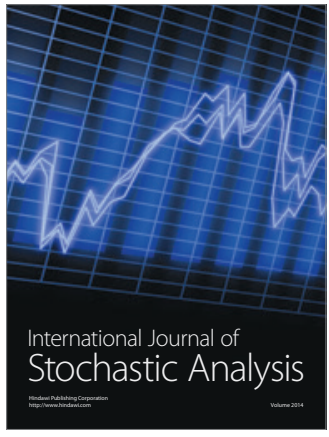

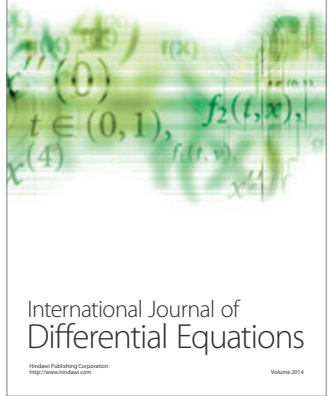
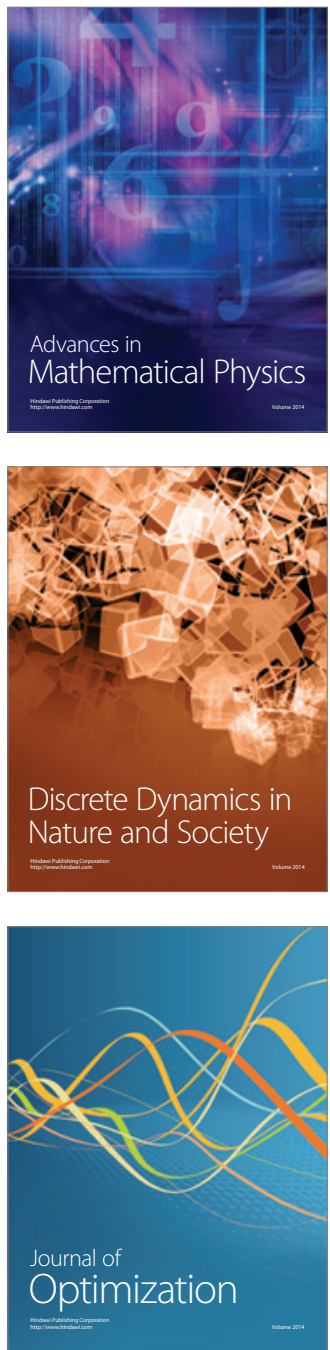